\title{
Oxidative stress in triazine pesticide toxicity: a review of the main biomarker findings
}

\author{
Tanja Živković Semren, Suzana Žunec, and Alica Pizent \\ Institute for Medical Research and Occupational Health, Zagreb, Croatia
}

[Received in February 2018; Similarity Check in February 2018; Accepted in June 2018]

\begin{abstract}
This review article provides a summary of the studies relying on oxidative stress biomarkers (lipid peroxidation and antioxidant enzymes in particular) to investigate the effects of atrazine and terbuthylazine exposure in experimental animals and humans published since 2010. In general, experimental animals showed that atrazine and terbuthylazine exposure mostly affected their antioxidant defences and, to a lesser extent, lipid peroxidation, but the effects varied by the species, sex, age, herbicide concentration, and duration of exposure. Most of the studies involved aquatic organisms as useful and sensitive bio-indicators of environmental pollution and important part of the food chain. In laboratory mice and rats changes in oxidative stress markers were visible only with exposure to high doses of atrazine. Recently, our group reported that low-dose terbuthylazine could also induce oxidative stress in Wistar rats. It is evident that any experimental assessment of pesticide toxic effects should take into account a combination of several oxidative stress and antioxidant defence biomarkers in various tissues and cell compartments. The identified effects in experimental models should then be complemented and validated by epidemiological studies. This is important if we wish to understand the impact of pesticides on human health and to establish safe limits.
\end{abstract}

KEY WORDS: atrazine; antioxidant enzymes; lipid peroxidation; terbuthylazine

The extensive use of pesticides in agriculture, public health, commerce, and individual households throughout the world speaks a lot about the importance of these compounds, but also raises concern about the risks involved for the environment, wildlife, and human health. Beside direct exposure of individuals who apply pesticides in agricultural, occupational, or residential settings, people are exposed to pesticides and their degradation products indirectly through water, air, dust, and food. These indirect routes generally involve low-level, yet long-term exposure (1). An increasing number of epidemiological studies have been suggesting that current levels of exposure are associated with risks to human health, including chronic diseases, cancers, neurological deficits, birth defects, and reproductive disorders $(2,3)$.

Pesticides can produce adverse physiological or biological effects, with a variety of biochemical changes at the molecular, cellular, or tissue level. These biochemical changes can be used as biological markers of effects in epidemiological studies as well as in animal toxicology studies. The most common biomarkers used to assess pesticide effects are related to DNA and RNA damage, modulation of gene expression, and oxidative stress (2). Although the underlying mechanisms are not known, a coherent body of evidence indicates that some pesticides, including organophosphates, organochlorines, organofluorines, carbamates, pyrethroids, bipyridyl

Correspondence to: Suzana Žunec, PhD, Toxicology Unit, Institute for Medical Research and Occupational Health, Ksaverska cesta 2, HR-10001 Zagreb, Croatia, E-mail: suzana@imi.hr herbicides, chloroacetanilide herbicides, and triazine herbicides can induce oxidative stress (4-6).

Biomarkers of oxidative stress have been demonstrated to be sensitive enough to high and low pesticide exposure alike. Thanks to oxidative stress biomarkers like malondialdehyde, F2-isoprostanes, thiobarbituric reactive substances, catalase and superoxide dismutase activities, 8-oxo- or 8-OH-deoxyguanosine, molecular epidemiological studies have provided us with evidence that oxidative stress is one of the key links between pesticides toxicity and multi-stage carcinogenesis (7-10). Furthermore, field studies in aquatic organisms confirm that oxidative stress is generated by exposure to several classes of pesticides (organochlorines, organofluorines, organophosphates, carbamates, pyrethroids, bipyridyl herbicides, triazine herbicides, and chloroacetanilide herbicides) (11). Induction of oxidative stress has also been reported in test animals exposed to organophosphorus pesticides, $N$-methyl carbamates, organochlorines, pyrethroids, and triazines (12). However, most animal studies use high concentrations of pesticides that are not normally encountered in the environment.

Oxidative stress occurs when the antioxidant defence mechanisms are overwhelmed by reactive oxygen species (ROS) generated through exposure to pesticides (10). This so called redox imbalance (with excess ROS) can modify proteins and lipids, change the DNA structure, activate stress-induced transcription factors, and produce inflammatory cytokines. General pathways by which pesticides increase ROS levels are their oxidative 
metabolism by cytochrome P450 enzymes, generation of redox-active metabolites, and impairment of the electron transport cascades in mitochondria $(10,13)$. ROS react readily with lipids, proteins, carbohydrates, and nucleic acids, and in doing so affect the structure and function of cellular components. When free radicals attack unsaturated fatty acids in cell membranes that contain lipids, lipid peroxidation occurs. One of the most common markers of lipid peroxidation is malondialdehyde (MDA), formed as its product, which is often assayed with thiobarbituric acid (TBA) (14). However, MDA is also a known mutagen (15). Radical chain reaction of lipid peroxidation appears to be a continuous physiological process. This process, if out of control, can alter essential cell functions and lead to cell death (16). Antioxidant defence includes enzymes like superoxide dismutase (SOD), catalase (CAT), and glutathione peroxidase (GPx), and non-enzymatic systems like glutathione (17). SOD is the first step in the defence against oxidative stress, as it catalyses dismutation of two superoxide radicals $\left(\mathrm{O}_{2}^{-}\right)$into molecular oxygen $\left(\mathrm{O}_{2}\right)$ and hydrogen peroxide $\left(\mathrm{H}_{2} \mathrm{O}_{2}\right)$, which is then neutralised by the combined action of CAT and GPx in all vertebrates (18). These enzymes have coordinated action, but changes in their levels may tip the balance toward oxidative stress.

As no single biomarker is sensitive and specific enough to identify or predict all oxidative stress effects (19), the aim of this paper was to summarise the representative studies in which the main markers of oxidative stress (20) were found associated with pesticide exposure and to draw conclusions about their significance in effective risk characterisation of pesticides.

We limited our scope to the triazine herbicides, atrazine and terbuthylazine in particular, because they have been well-researched and widely used in the USA and Europe, respectively. Our review includes key published literature and regulatory reports as well as our own results obtained within the project Organic Pollutants in Environment Markers and Biomarkers of Toxicity (OPENTOX).

\section{Triazine herbicides}

Herbicides are the most widely used class of pesticides, accounting for more than $60 \%$ of all pesticides applied in agriculture (21). They aim to inhibit biochemical and physiological processes that regulate plant growth. Their target sites are usually enzymes involved in the primary metabolic pathways or proteins carrying out essential physiological functions in plants (22). The main mechanism of triazine herbicides action is the inhibition of photosynthesis. Triazines bind to the QB protein in the photosystem II reaction centre and block the flow of electrons through the photosynthetic electron transport chain (23). Since they provide application flexibility, triazines are extensively used in conservation tillage programs as part of sustainable agriculture and are very effective in controlling many weeds resistant to other herbicides (24).Today, one or more of the triazine herbicides are marketed in more than 100 countries around the world (24). Although several classes of triazine herbicides have been developed since the 1950s, chloro-s-triazines have been a mainstay of preemergent application.

Evaluation of triazine herbicides revealed that these are chemicals with low toxic potential for humans; however, there are many controversies on this issue. For instance, in the beginning of the 2000s science and regulatory reviews conducted by the United Kingdom for the European Union (25-27), the Australian Pesticides and Veterinary Medicines Authority $(28,29)$, the International Agency for Research on Cancer (30), and the US Environmental Protection Agency (EPA) $(31,32)$ all supported the safety and continued availability of atrazine for weed control. Atrazine is still the most prevalent corn crop pesticide in the US, but in the EU it has been banned since 2004. The European Commission decided to ban atrazine not because of any specific toxicological reasons but because it was concerned that its residues in groundwater might exceed its nominal limit of $0.1 \mu \mathrm{g} \mathrm{L}^{-1}$ (33). New studies about atrazine's toxicological profile (acute and chronic effects, reproductive and developmental toxicity, mutagenicity, immunotoxicity) and environmental risks brought changes in regulatory positions. According to Dikshith (34), atrazine and associated compounds are of particular concern for human health and the environment due to: 1) evidence of carcinogenicity, reproductive/developmental effects, organ toxicity, immune system effects, and genotoxicity to humans; 2) likely adverse effects on non-target plants and animals, especially on the reproductive and developmental function in aquatic and amphibious species; and 3) extensive contamination of surface, ground, and drinking water.

\section{INVOLVEMENT OF OXIDATIVE STRESS IN ATRAZINE TOXICITY}

Atrazine [(6-chloro- $N$-ethyl-N0-(1-methylethyl)-1,3,5triazine-2,4-diamine] has been extensively studied and reviewed because of its moderate persistence in the environment and contamination of surface, ground, and drinking water that has raised a number of health concerns (35-38). Atrazine is generally considered an endocrine disruptor with adverse effects on the endocrine, central nervous, and immune system (39). Even though some disagree that it disrupts the endocrine system, a number of adverse effects have been reported, especially on the reproductive systems of rats, pigs, fish, and amphibians (39). Atrazine has been reported to cause mammary gland tumours in female Sprague-Dawley rats, which implies that it may be carcinogenic (40). Case-control studies, however, showed only weak associations between atrazine and non-Hodgkin lymphoma, ovarian, and prostate cancer (41-44). Based on the available animal and human data, the International Agency for Research on Cancer (IARC) (30) and the US EPA(31) have characterised atrazine as "not likely to be carcinogenic in humans". 
However, the atrazine effects in humans are not fully understood. There are ongoing studies looking for molecular evidence of atrazine's adverse effects, especially at environmental levels. Yet, there is also a coherent body of evidence that atrazine induced oxidative stress. Table 1 summarises the findings of the studies published from 2010 till now, which evidence changes in the main oxidative stress biomarkers.

We start with aquatic organisms first, as they have proven their usefulness as experimental models for the evaluation of adverse effects of atrazine. Tested atrazine concentrations ranged from $0.3 \mu \mathrm{g} \mathrm{L}^{-1}$, which is the highest environmental concentration reported in Czech rivers $(45,46,54)$ and $4.28 \mu \mathrm{g} \mathrm{L}^{-1}$, which is $1 / 5$ of the 96 -hour $\mathrm{LC}_{50}$ for common carp (Cyprinus carpio) $(49,50)$, to concentrations as high as hundred or thousand $\mu \mathrm{g} \mathrm{L}^{-1}$. Atrazine did not have any influence on the levels of lipid peroxidation products in aquatic organisms at early stages of development (45-48). In adult aquatic organisms, increases in MDA or TBARS were reported after sub-chronic exposure (14-40 days) to higher atrazine concentrations $\left(\geq 42.8 \mu \mathrm{g} \mathrm{L}^{-1}\right)(49-52,54)$, whereas acute exposure of one or two days did not cause lipid peroxidation $(56,62)$. An exception is the study by Owolabi et al. (60), who reported higher MDA concentrations after a four-day atrazine exposure.

As for atrazine effects on antioxidant enzyme activities, sub-chronic exposure to an environmentally relevant concentration of atrazine $\left(0.3 \mu \mathrm{g} \mathrm{L}^{-1}\right)$ caused an increase in the activity of CAT, GPx, GST, and SOD in common carp embryos and larvae, whereas sub-chronic exposure to environmentally relevant concentration of atrazine-2-hydroxy $\left(0.66 \mu \mathrm{g} \mathrm{L}^{-1}\right) \mathrm{did}$ not affect their activities (46). In adult carp, several studies reported that a 40-day exposure to atrazine in concentrations above $42.8 \mu \mathrm{g} \mathrm{L}^{-1}$ resulted in a decrease in CAT, GPx, and SOD activities (49-51).

In adult zebrafish (Danio rerio), a 28-day exposure to low atrazine concentrations, including the environmentally relevant $0.3 \mu \mathrm{g} \mathrm{L}^{-1}$ caused a decrease in CAT (54), but exposure to higher concentrations of atrazine $\left(>100 \mu \mathrm{g} \mathrm{L}^{-1}\right)$ for 14 or 21 days resulted in an increase in CAT and SOD activities in the liver homogenate $(52,53)$.

In other fish species, sub-chronic exposure to low atrazine increased CAT and SOD activities $(56,59)$, whereas acute exposure generally lowered CAT, GPx, and SOD activities $(55,57,58)$, with exception of neotropical fish, which showed no change (56). Furthermore, in two studies atrazine exposure of Gammarus kischineffensis and shrimp Palaemonetes argentinus resulted in lower CAT but higher SOD activity (61, 62).

Two studies in drosophilids observed developmental and genotoxic effects $(81,82)$, which prompted Figuera et al. (63) to investigate if these effects were owed to oxidative stress. To do that, they exposed the embryos (newly fertilised eggs) of the fruit fly Drosophila melanogaster to atrazine concentrations ranging between 10 and $100 \mu \mathrm{mol} \mathrm{L}^{-1}$ through diet until they developed into adult flies. While the larvae showed no changes in ROS levels, the adult flies did, so the authors concluded that redox imbalance must have been related to changes in metabolism after metamorphosis. Generally, female flies were more sensitive to oxidative stress; they showed increased ROS levels and antioxidant capacity against peroxyl radicals (ACAP) at atrazine concentration of $10 \mu \mathrm{mol} \mathrm{L}{ }^{-1}$, while LPO was did not increase until $100 \mu \mathrm{mol} \mathrm{L}^{-1}$.

The growing evidence of recent declines in pollinator populations has raised great concern about economic and environmental consequences of these declines. One outstanding threat to honeybees (Apis mellifera) is the nontarget toxicity of pesticides. Honeybees and other pollinators are exposed to atrazine and other herbicides through pollen, nectar, water, and dust, particularly in the vast areas with herbicide-treated monoculture crops. One study (64) investigated lipid peroxidation in caged honeybees exposed to syrup spiked with realistic environmental doses of atrazine (1.25 to $5 \mathrm{ng}^{-1}$ per bee) and found no significant changes in their TBARS levels. In another study (65), honeybees in a laboratory were exposed to atrazine concentrations ranging from 0.1 to $10 \mu \mathrm{g} \mathrm{L}^{-1}$ for 24 hours, whereas hives in the field were treated with $10 \mu \mathrm{g} \mathrm{L}^{-1}$ of atrazine a day for 28 days. GPx and GST activities dropped, whereas MDA levels increased in both the laboratory and hive honeybees.

Rodent studies have made a big contribution to the knowledge about the involvement of oxidative stress in the toxicity mechanisms of atrazine. Table 1 sums up the findings of several important studies on mice and rats, which reported changes in the main oxidative stress biomarkers. For instance, mice receiving $78.25 \mathrm{mg} \mathrm{kg}^{-1}$ bw $\left(1 / 8 \mathrm{LD}_{50}\right)$ of atrazine intraperitoneally every other day for 14 days had higher liver MDA and GST and lower GSH, antioxidant power (FRAP), SOD, and CAT. The latter two were also low in the kidney tissue (66). Similar effects were noticed in mice receiving 100 , 200 , or $400 \mathrm{mg} \mathrm{kg}^{-1}$ bw of atrazine by gavage every day for 21 days (67).

In the studies conducted on rats, the animals were exposed to atrazine acutely ( 7 to 14 days) or subchronically (15 to 52 days). The maximum tolerated dose of atrazine in rats, according to Singh et al. (68) and Narotsky et al. (83) is $300 \mathrm{mg} \mathrm{kg}^{-1}$ bw or $1 / 10$ of its oral $\mathrm{LD}_{50}$. In a number of studies (summarised in Table 1), sub-chronic exposure to $300 \mathrm{mg} \mathrm{kg}^{-1}$ of atrazine resulted in higher MDA levels in the liver and adrenal cortex of rats $(70,72,79)$. Higher MDA was also detected in the testes, epididymis, brain, liver, and kidney of rats exposed to $25-200 \mathrm{mg} \mathrm{kg}^{-1}$ of atrazine $(69,73,76,80)$. As for the antioxidant defences in rats, higher atrazine doses (300 m k kg ${ }^{-1}$ ) increased the activities of CAT, SOD, GPX, and GST and decreased GSH levels in blood and liver $(68,70,72)$, whereas all of these markers dropped in the adrenal cortex (79). At doses from 25 to $200 \mathrm{mg} \mathrm{kg}^{-1}$, atrazine lowered CAT activity in rat testis, epididymis, brain, liver, and kidney (69, $71,73,76,80)$.

In contrast, oral exposure to low atrazine doses $\left(0.3 \mathrm{mg} \mathrm{kg}^{-1}\right.$ or $12.5 \mathrm{mg} \mathrm{kg}^{-1}$ ) did not change MDA in rat liver, kidney, and testis $(74,75)$ or disturb antioxidant defences by changing 
Table 1 Changes in oxidative stress markers in experimental models after exposure to atrazine

\begin{tabular}{|c|c|c|c|c|c|}
\hline Experimental model & $\begin{array}{c}\text { Route of } \\
\text { administration }\end{array}$ & $\begin{array}{l}\text { Exposure } \\
\text { duration }\end{array}$ & $\begin{array}{l}\text { Concentration } \\
/ \text { dose }\end{array}$ & $\begin{array}{c}\text { Oxidative stress } \\
\text { markers }\end{array}$ & Ref. \\
\hline $\begin{array}{l}\text { Fish - common carp, } \\
\text { embryos and larvae }\end{array}$ & water & 36 days & $\begin{array}{l}0.66 \mu \mathrm{g} \mathrm{L}{ }^{-1} \\
\text { (Atrazine } \\
\text { 2-hydroxy) }\end{array}$ & $\begin{array}{l}\bullet \mathrm{CAT} \\
\bullet \mathrm{GR} \\
\bullet \mathrm{GSH} \\
\bullet \mathrm{GST} \\
\bullet \mathrm{SOD} \\
\bullet \mathrm{TBARS}\end{array}$ & $\begin{array}{l}\text { Velisek et al. } \\
\text { (45) }\end{array}$ \\
\hline $\begin{array}{l}\text { Fish - common carp, } \\
\text { embryos and larvae }\end{array}$ & water & 33 days & $\begin{array}{c}0.3 \mu \mathrm{g} \mathrm{L}^{-1} \\
30 \mu \mathrm{g} \mathrm{L}^{-1} \\
100 \mu \mathrm{g} \mathrm{L}^{-1} \\
300 \mu \mathrm{g} \mathrm{L}^{-1}\end{array}$ & $\begin{array}{c}\uparrow \mathrm{CAT}\left(0.3 \mu \mathrm{g} \mathrm{L}^{-1}\right) \\
\uparrow \mathrm{GPX} \\
\bullet \mathrm{GR} \\
\uparrow \mathrm{GST}\left(0.3 \mu \mathrm{g} \mathrm{L}^{-1}\right) \\
\uparrow \mathrm{SOD}\left(0.3 \mu \mathrm{g} \mathrm{L}^{-1}\right) \\
\downarrow \text { SOD }\left(300 \mu \mathrm{g} \mathrm{L}^{-1}\right) \\
\bullet \text { TBARS }\end{array}$ & $\begin{array}{l}\text { Chromcova } \\
\text { et al. }(46)\end{array}$ \\
\hline Marbled crayfish eggs & water & 77 days & $\begin{array}{c}0.66 \mu \mathrm{g} \mathrm{L}-1 \\
\text { (Atrazine } \\
\text { 2-hydroxy) }\end{array}$ & $\begin{array}{c}\uparrow C A T \\
\bullet \text { GR } \\
\bullet \text { SOD } \\
\bullet \text { TBARS }\end{array}$ & $\begin{array}{l}\text { Velisek et al. } \\
\text { (47) }\end{array}$ \\
\hline Fish - zebrafish embryos & water & 4 days & $0.1 \mathrm{mmol} \mathrm{L}^{-1}$ & $\begin{array}{l}\bullet \text { CAT } \\
\bullet \text { GPx } \\
\downarrow \text { GSH } \\
\text { •TBARS }\end{array}$ & $\begin{array}{l}\text { Adeyemi et } \\
\text { al. (48) }\end{array}$ \\
\hline Fish - common carp & water & 40 days & $\begin{array}{l}4.28 \mu \mathrm{g} \mathrm{L}^{-1} \\
42.8 \mu \mathrm{g} \mathrm{L}^{-1} \\
428 \mu \mathrm{g} \mathrm{L}^{-1}\end{array}$ & $\begin{array}{c}\uparrow \mathrm{CAT}\left(4.28 \mu \mathrm{g} \mathrm{L}^{-1} \text {-liver }\right. \\
\text { and gill) } \\
\downarrow \mathrm{CAT}\left(42.8,428 \mu \mathrm{gL}^{-1} \text {-liver; }\right. \\
428 \mu \mathrm{g} \mathrm{L} \mathrm{L}^{-1} \text {-gill) } \\
\downarrow \mathrm{GPx} \text { (liver; } 42.8, \\
428 \mu \mathrm{g} \mathrm{L}^{-1} \text {-gill) } \\
\downarrow \mathrm{SOD}\left(42.8,428 \mu \mathrm{g} \mathrm{L}^{-1}\right. \\
\text {-liver and gill) } \\
\uparrow \mathrm{MDA}\left(42.8,428 \mu \mathrm{gL}^{-1}\right. \\
\text {-liver, } 428 \mu \mathrm{gL}^{-1} \text {-gill) }\end{array}$ & $\begin{array}{l}\text { Xing et al. } \\
\quad(49)\end{array}$ \\
\hline Fish - common carp & water & 40 days & $\begin{array}{l}4.28 \mu \mathrm{g} \mathrm{L}^{-1} \\
42.8 \mu \mathrm{g} \mathrm{L}^{-1} \\
428 \mu \mathrm{g} \mathrm{L}^{-1}\end{array}$ & $\begin{array}{c}\downarrow \text { CAT (428 } \mu \mathrm{g} \mathrm{L}^{-1} \text {-brain } \\
\text { and kidney) } \\
\uparrow \mathrm{GPx}\left(428 \mu \mathrm{g} \mathrm{L}{ }^{-1}-\right. \\
\text { kidney) } \\
\downarrow \text { GPx } \\
\left(42.8,428 \mu \mathrm{g} \mathrm{L}^{-1} \text {-brain; }\right. \\
428 \mu \mathrm{g} \mathrm{L}-1 \text {-kidney) } \\
\downarrow \mathrm{SOD}(42.8,428 \mu \mathrm{g} \mathrm{L}-1 \\
\text {-brain and kidney) } \\
\uparrow \mathrm{MDA}^{-1} \\
\text { (428 } \mu \mathrm{g} \mathrm{L}^{-1} \text {-brain; } 42.8, \\
428 \mu \mathrm{g} \mathrm{L}^{-1} \text {-kidney) }\end{array}$ & $\begin{array}{l}\text { Xing et al. } \\
\quad(50)\end{array}$ \\
\hline Fish - common carp & water & 40 days & $\begin{array}{l}4.28 \mu \mathrm{g} \mathrm{L}^{-1} \\
42.8 \mu \mathrm{g} \mathrm{L}^{-1} \\
428 \mu \mathrm{g} \mathrm{L}^{-1}\end{array}$ & $\begin{array}{c}\downarrow \text { SOD } \\
\text { (42.8, } 428 \mu \mathrm{g} \mathrm{L} \text {-1 }^{-} \text {-spleen, } \\
\text { head, kidney) } \\
\uparrow \mathrm{MDA}^{-1} \\
\left(42.8,428 \mu \mathrm{gL} \mathrm{L}^{-1} \text {-spleen; }\right. \\
428 \mu \mathrm{g} \mathrm{L}^{-1} \text {-head kidney) }\end{array}$ & $\begin{array}{l}\text { Wang et al. } \\
\qquad(51)\end{array}$ \\
\hline
\end{tabular}




\begin{tabular}{|c|c|c|c|c|c|}
\hline Experimental model & $\begin{array}{c}\text { Route of } \\
\text { administration }\end{array}$ & $\begin{array}{l}\text { Exposure } \\
\text { duration }\end{array}$ & $\begin{array}{c}\text { Concentration } \\
\text { / dose }\end{array}$ & $\begin{array}{c}\begin{array}{c}\text { Oxidative stress } \\
\text { markers }\end{array} \\
\end{array}$ & Ref. \\
\hline Adult female fish zebrafish & water & 14 days & $\begin{array}{c}1 \mu \mathrm{g} \mathrm{L}^{-1} \\
10 \mu \mathrm{g} \mathrm{L}^{-1} \\
100 \mu \mathrm{g} \mathrm{L}^{-1} \\
1000 \mu \mathrm{g} \mathrm{L}^{-1}\end{array}$ & $\begin{array}{c}\uparrow \mathrm{CAT}\left(1000 \mu \mathrm{g} \mathrm{L}^{-1} \text {-liver: }\right. \\
\left.10 \mu \mathrm{g} \mathrm{L}^{-1} \text {-ovary }\right) \\
\downarrow \mathrm{GSH}\left(10,100,1000 \mu \mathrm{gL}^{-1}\right. \\
\text {-liver, } 1,100,1000 \mu \mathrm{gL}^{-1} \\
\text {-ovary) } \\
\uparrow \mathrm{SOD}\left(100,1000 \mu \mathrm{gL} \mathrm{L}^{-1}\right. \\
\left.\text {-liver; } 10 \mu \mathrm{gL} \mathrm{L}^{-1} \text {-ovary }\right) \\
\uparrow \mathrm{MDA}\left(100,1000 \mu \mathrm{gL}^{-1}\right. \\
\text {-liver })\end{array}$ & $\begin{array}{l}\text { Jin et al. } \\
\text { (52) }\end{array}$ \\
\hline Fish - zebrafish & water & 21 days & $\begin{array}{c}2500 \mu \mathrm{g} \mathrm{L}^{-1} \\
5000 \mu \mathrm{g} \mathrm{L}^{-1} \\
10000 \mu \mathrm{g} \mathrm{L}^{-1}\end{array}$ & $\begin{array}{l}\uparrow \mathrm{CAT}\left(5000 \mu \mathrm{g} \mathrm{L}^{-1}\right) \\
\uparrow \operatorname{SOD}\left(2500 \mu \mathrm{g} \mathrm{L}^{-1}\right)\end{array}$ & $\begin{array}{l}\text { Zhu et al. } \\
\quad(53)\end{array}$ \\
\hline Fish - zebrafish & water & 28 days & $\begin{array}{c}0.3 \mu \mathrm{g} \mathrm{L}^{-1} \\
3 \mu \mathrm{g} \mathrm{L}^{-1} \\
30 \mu \mathrm{g} \mathrm{L}^{-1} \\
90 \mu \mathrm{g} \mathrm{L}^{-1}\end{array}$ & $\begin{array}{c}\downarrow \mathrm{CAT} \\
\uparrow \mathrm{GPx}\left(30 \mu \mathrm{g} \mathrm{L}^{-1}\right) \\
\uparrow \mathrm{GR}\left(0.3,30 \mu \mathrm{g} \mathrm{L}^{-1}\right) \\
\downarrow \mathrm{GST}\left(90 \mu \mathrm{g} \mathrm{L}^{-1}\right) \\
\bullet \mathrm{SOD} \\
\uparrow \operatorname{TBARS}\left(30,90 \mu \mathrm{g} \mathrm{L}^{-1}\right)\end{array}$ & $\begin{array}{l}\text { Blahová et } \\
\text { al. (54) }\end{array}$ \\
\hline Fish - zebrafish & water & 1 day & $300 \mu \mathrm{g} \mathrm{L}^{-1}$ & $\begin{array}{c}\downarrow \text { CAT (liver) } \\
\downarrow \text { GPx (liver, brain) } \\
\uparrow \text { GSH (kidney) } \\
\downarrow \text { SOD (kidney) } \\
\bullet \text { MDA (liver, kidney, } \\
\text { brain) }\end{array}$ & $\begin{array}{l}\text { Shukla et al. } \\
\quad(55)\end{array}$ \\
\hline $\begin{array}{l}\text { Neotropical fish } \\
\text { (Prochilodus lineatus) }\end{array}$ & water & $\begin{array}{c}\text { Acute }(2 \\
\text { days) and } \\
\text { subchronic } \\
\text { (14 days) }\end{array}$ & $\begin{array}{l}2 \mu \mathrm{g} \mathrm{L}^{-1} \\
10 \mu \mathrm{g} \mathrm{L}^{-1} \\
25 \mu \mathrm{g} \mathrm{L}^{-1}\end{array}$ & $\begin{array}{c}\text { Subchronic } \\
\uparrow \mathrm{CAT}\left(10 \mu \mathrm{g} \mathrm{L}{ }^{-1}\right) \\
\bullet \mathrm{GSH} \\
\bullet \mathrm{GPx} \\
\uparrow \mathrm{GST}\left(10 \mu \mathrm{g} \mathrm{L}^{-1}\right) \\
\uparrow \mathrm{SOD}\left(10 \mu \mathrm{g} \mathrm{L}^{-1}\right) \\
\uparrow \mathrm{LPO}\left(10 \mu \mathrm{g} \mathrm{L}^{-1}\right)\end{array}$ & $\begin{array}{l}\text { Paulino et } \\
\text { al. (56) }\end{array}$ \\
\hline $\begin{array}{l}\text { Neotropical fish } \\
\text { (Prochilodus lineatus) }\end{array}$ & water & $\begin{array}{c}1 \text { and } 2 \\
\text { days }\end{array}$ & $\begin{array}{l}2 \mu \mathrm{g} \mathrm{L}^{-1} \\
10 \mu \mathrm{g} \mathrm{L}^{-1}\end{array}$ & $\begin{array}{c}\downarrow \mathrm{CAT} \\
\downarrow \mathrm{GPx} \\
\uparrow \mathrm{GR} \\
\downarrow \mathrm{GSH}\left(2^{\text {nd }} \text { day }\right) \\
\downarrow \text { SOD } \\
\downarrow \mathrm{MDA}\left(1^{\text {st }} \text { day } ; 2 \mu \mathrm{g} \mathrm{L}^{-1}-\right. \\
\left.2^{\text {nd }} \text { day }\right) \\
\downarrow \mathrm{ROS}^{-}\left(1^{\text {st }} \text { day; }\right. \\
\left.10 \mu \mathrm{g} \mathrm{L}^{-1}-2^{\text {nd }} \text { day }\right)\end{array}$ & $\begin{array}{l}\text { Santos et al. } \\
\quad(57)\end{array}$ \\
\hline $\begin{array}{l}\text { Neotropical catfish } \\
\text { (Rhamdia quelen) }\end{array}$ & water & 4 days & $\begin{array}{c}2 \mu \mathrm{g} \mathrm{L}^{-1} \\
10 \mu \mathrm{g} \mathrm{L}^{-1} \\
100 \mu \mathrm{g} \mathrm{L}^{-1}\end{array}$ & $\begin{array}{c}\downarrow \mathrm{CAT} \\
\downarrow \mathrm{GPx} \\
\downarrow \mathrm{GR} \\
\downarrow \mathrm{GSH}\left(100 \mu \mathrm{g} \mathrm{L}^{-1}\right) \\
\downarrow \text { GST } \\
\bullet \mathrm{SOD} \\
\bullet \text { LPO }\end{array}$ & $\begin{array}{l}\text { Mela et al. } \\
\quad(58)\end{array}$ \\
\hline $\begin{array}{l}\text { Fish - Channa punctatus } \\
\text { (Bloch) }\end{array}$ & water & 15 days & $\begin{array}{c}4238 \mu \mathrm{g} \mathrm{L}^{-1} \\
5300 \mu \mathrm{g} \mathrm{L}^{-1} \\
10600 \mu \mathrm{g} \mathrm{L}^{-1}\end{array}$ & $\begin{array}{c}\uparrow \text { CAT (till day } 7 \text { ) } \\
\downarrow \text { CAT (after day 7) } \\
\uparrow \text { GR (after day 10) } \\
\uparrow \text { SOD } \\
\uparrow \text { TBARS }\end{array}$ & $\begin{array}{l}\text { Nwani et al. } \\
\text { (59) }\end{array}$ \\
\hline
\end{tabular}




\begin{tabular}{|c|c|c|c|c|c|}
\hline Experimental model & $\begin{array}{c}\text { Route of } \\
\text { administration }\end{array}$ & $\begin{array}{l}\text { Exposure } \\
\text { duration }\end{array}$ & $\begin{array}{l}\text { Concentration } \\
\text { / dose }\end{array}$ & $\begin{array}{c}\begin{array}{c}\text { Oxidative stress } \\
\text { markers }\end{array} \\
\end{array}$ & Ref. \\
\hline $\begin{array}{l}\text { African catfish (Clarias } \\
\text { gariepinus) }\end{array}$ & water & $\begin{array}{l}\text { Acute }(4 \\
\text { days) } \\
\text { and } \\
\text { Chronic } \\
\text { (28 days) }\end{array}$ & $\begin{array}{c}\text { Acute (4 days) } \\
28 \mu \mathrm{g} \mathrm{L}^{-1} \\
30 \mu \mathrm{g} \mathrm{L}^{-1} \\
32 \mu \mathrm{g} \mathrm{L}^{-1} \\
34 \mu \mathrm{g} \mathrm{L}^{-1} \\
\text { Chronic (28 } \\
\text { days) } \\
7 \mu \mathrm{g} \mathrm{L}^{-1} \\
7.50 \mu \mathrm{g} \mathrm{L}^{-1} \\
8 \mu \mathrm{g} \mathrm{L}^{-1} \\
8.50 \mu \mathrm{g} \mathrm{L}^{-1}\end{array}$ & $\begin{array}{c}\text { Acute } \\
\downarrow \text { SOD }\left(28,30,34 \mu \mathrm{g} \mathrm{L}^{-1} \text { - }\right. \\
\text { blood) } \\
\uparrow \mathrm{SOD} \text { (gill and liver) } \\
\uparrow \mathrm{MDA}\left(30,32 \mu \mathrm{g} \mathrm{L}^{-1} \text { - }\right. \\
\text { blood; gill and liver) } \\
\text { Chronic } \\
\downarrow \text { SOD }\left(7,7.5,8 \mu \mathrm{g} \mathrm{L}^{-1}\right. \\
\text {-blood; } 7.5,8, \\
\left.8.5 \mu \mathrm{g} \mathrm{L} \mathrm{L}^{-1} \text {-gill; liver }\right) \\
\uparrow \mathrm{SOD}\left(8.5 \mu \mathrm{g} \mathrm{L} \mathrm{L}^{-1} \text {-blood) }\right. \\
\uparrow \mathrm{MDA}\left(7.5,8 \mu \mathrm{g} \mathrm{L}^{-1}\right. \\
\text {-blood; gill and liver) }\end{array}$ & $\begin{array}{l}\text { Owolabi et } \\
\text { al. (60) }\end{array}$ \\
\hline Gammarus kischineffensis & water & $\begin{array}{l}1,2,3,4 \\
\text { days }\end{array}$ & $\begin{array}{c}1000- \\
50,000 \mu \mathrm{g} \mathrm{L}^{-1}\end{array}$ & $\begin{array}{c}\downarrow \text { CAT (after } 3 \text { days) } \\
\bullet \text { CAT (after } 4 \text { days) } \\
\uparrow \text { GST } \\
\downarrow \text { GR } \\
\uparrow \text { SOD }\end{array}$ & $\begin{array}{l}\text { Demirci et } \\
\text { al. (61) }\end{array}$ \\
\hline $\begin{array}{l}\text { Shrimp (Palaemonetes } \\
\text { argentinus) }\end{array}$ & water & 1 day & $400 \mu \mathrm{g} \mathrm{L}^{-1}$ & $\begin{array}{c}\uparrow \mathrm{GR} \\
\uparrow \mathrm{GST}(\text { cytosolic) } \\
\bullet \mathrm{GST} \text { (microsomal) } \\
\uparrow \mathrm{SOD} \\
\bullet \text { TBARS }\end{array}$ & $\begin{array}{l}\text { Griboff et al. } \\
\quad(62)\end{array}$ \\
\hline $\begin{array}{l}\text { Fly - Drosophila } \\
\text { melanogaster, embryo- } \\
\text { larval stage, wild type } \\
\text { strains }\end{array}$ & $\begin{array}{l}\text { experimental } \\
\text { medium }\end{array}$ & $\begin{array}{l}\text { The } \\
\text { embryos } \\
\text { were } \\
\text { allowed to } \\
\text { develop } \\
\text { until } \\
\text { emergence }\end{array}$ & $\begin{array}{l}0.01 \mathrm{mmol} \mathrm{L}^{-1} \\
0.1 \mathrm{mmol} \mathrm{L}^{-1}\end{array}$ & 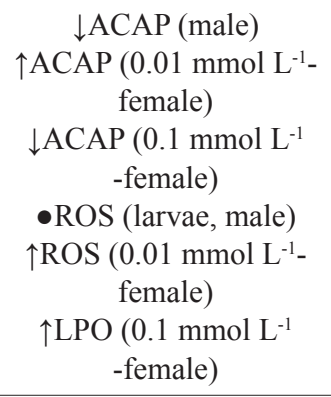 & $\begin{array}{l}\text { Figueira et } \\
\text { al. (63) }\end{array}$ \\
\hline Honeybees & food & 10 days & $\begin{array}{l}1.25 \mathrm{ng} \text { per bee } \\
2.50 \mathrm{ng} \text { per bee } \\
5.0 \mathrm{ng} \text { per bee }\end{array}$ & •TBARS & $\begin{array}{l}\text { Helmer et } \\
\text { al. (64) }\end{array}$ \\
\hline Honeybees & food & 1 day & $\begin{array}{l}0.1 \mu \mathrm{g} \mathrm{L}^{-1} \\
1 \mu \mathrm{g} \mathrm{L}^{-1} \\
10 \mu \mathrm{g} \mathrm{L}^{-1}\end{array}$ & $\begin{array}{c}\downarrow \mathrm{GPx} \\
\left(1,10 \mu \mathrm{g} \mathrm{L}^{-1}\right) \\
\downarrow \mathrm{GSH} \\
\downarrow \mathrm{GST}\left(1,10 \mu \mathrm{g} \mathrm{L}^{-1}\right) \\
\uparrow \mathrm{MDA} \\
\downarrow \text { TAA }\end{array}$ & $\begin{array}{l}\text { Williams } \\
\text { (65) }\end{array}$ \\
\hline Male mice & intraperitoneally & 14 days & $78.25 \mathrm{mg} \mathrm{kg}^{-1}$ & $\begin{array}{c}\downarrow \text { CAT (liver, kidney) } \\
\downarrow \text { GSH (liver) } \\
\uparrow \text { GST (liver) } \\
\downarrow \text { SOD (liver, kidney) } \\
\uparrow \text { MDA (liver) } \\
\downarrow \text { FRAP (liver) }\end{array}$ & $\begin{array}{l}\text { EL- } \\
\text { Shenawy et } \\
\text { al. (66) }\end{array}$ \\
\hline $\begin{array}{l}\text { Male and female Balb/c } \\
\text { mice }\end{array}$ & gavage & 21 days & $\begin{array}{l}100 \mathrm{mg} \mathrm{kg}^{-1} \\
200 \mathrm{mg} \mathrm{kg}^{-1} \\
400 \mathrm{mg} \mathrm{kg}^{-1}\end{array}$ & $\begin{array}{c}\downarrow \mathrm{GSH} \\
\uparrow \mathrm{GSSG} \\
\uparrow \mathrm{ROS}\end{array}$ & $\begin{array}{l}\text { Gao et al. } \\
\quad(67)\end{array}$ \\
\hline
\end{tabular}




\begin{tabular}{|c|c|c|c|c|c|}
\hline Experimental model & $\begin{array}{c}\text { Route of } \\
\text { administration }\end{array}$ & $\begin{array}{l}\text { Exposure } \\
\text { duration }\end{array}$ & $\begin{array}{c}\text { Concentration } \\
\text { / dose }\end{array}$ & $\begin{array}{c}\text { Oxidative stress } \\
\text { markers }\end{array}$ & Ref. \\
\hline Male Wistar rats & gavage & $\begin{array}{l}7,14 \text { and } \\
21 \text { days }\end{array}$ & $300 \mathrm{mg} \mathrm{kg}^{-1}$ & $\begin{array}{c}\uparrow \mathrm{CAT} \\
\uparrow \mathrm{GPx} \text { (after } 14 \text { and } 21 \\
\text { days) } \\
\downarrow \text { GSH } \\
\uparrow \mathrm{GST} \\
\uparrow \mathrm{SOD} \text { (after } 14 \text { and } 21 \\
\text { days) }\end{array}$ & $\begin{array}{l}\text { Singh et al. } \\
\text { (68) }\end{array}$ \\
\hline $\begin{array}{l}\text { Adult male } \\
\text { Wistar rats }\end{array}$ & gavage & 16 days & $\begin{array}{l}120 \mathrm{mg} \mathrm{kg}^{-1} \\
200 \mathrm{mg} \mathrm{kg}^{-1}\end{array}$ & $\begin{array}{c}\downarrow \text { CAT (epididymis) } \\
\bullet \text { GSH (testes and } \\
\text { epididymis) } \\
\downarrow \text { GST (testes and } \\
\text { epididymis) } \\
\downarrow \text { SOD (testes and } \\
\text { epididymis) } \\
\uparrow \text { MDA (testes and } \\
\text { epididymis) }\end{array}$ & $\begin{array}{l}\text { Abarikwu et } \\
\text { al. (69) }\end{array}$ \\
\hline Male Wistar rats & gavage & $\begin{array}{l}7,14 \text { and } \\
21 \text { days }\end{array}$ & $300 \mathrm{mg} \mathrm{kg}^{-1}$ & $\begin{array}{c}\uparrow \text { CAT (after } 14 \text { and } 21 \\
\text { days) } \\
\uparrow \text { GPx (after } 14 \text { and } 21 \\
\text { days) } \\
\downarrow \text { GSH } \\
\uparrow \text { GST } \\
\uparrow \text { SOD (after } 14 \text { and } 21 \\
\text { days) } \\
\uparrow \text { MDA }\end{array}$ & $\begin{array}{l}\text { Singh et al. } \\
\quad(70)\end{array}$ \\
\hline $\begin{array}{l}\text { Peripubertal male Wistar } \\
\text { rats }\end{array}$ & gavage & 27 days & $\begin{array}{l}50 \mathrm{mg} \mathrm{kg}^{-1} \\
200 \mathrm{mg} \mathrm{kg}^{-1}\end{array}$ & $\begin{array}{c}\downarrow \text { CAT (200 mg kg-1- } \\
\text { interstitial testicular } \\
\text { cells) } \\
\downarrow \text { GPx (200 mg kg-1- } \\
\text { interstitial testicular } \\
\text { cells) } \\
\uparrow \text { GPx (liver) } \\
\uparrow \text { GST (200 mg kg-1 } \\
\text {-liver) } \\
\downarrow \text { GST (interstitial } \\
\text { testicular cells) } \\
\bullet \text { SOD } \\
\bullet \text { TBARS }\end{array}$ & $\begin{array}{l}\text { Pogrmić- } \\
\text { Majkić et al. } \\
\text { (71) }\end{array}$ \\
\hline $\begin{array}{l}\text { Male } \\
\text { Wistar rats }\end{array}$ & & 14 days & $300 \mathrm{mg} \mathrm{kg}^{-1}$ & $\begin{array}{l}\uparrow \mathrm{CAT} \\
\uparrow \mathrm{GPx} \\
\uparrow \mathrm{GST} \\
\downarrow \mathrm{GSH} \\
\uparrow \mathrm{SOD} \\
\uparrow \mathrm{MDA}\end{array}$ & $\begin{array}{l}\text { Shirisha et } \\
\text { al. (72) }\end{array}$ \\
\hline Adult Wistar rats & $\begin{array}{c}\text { orally } \\
\text { administered }\end{array}$ & 16 days & $120 \mathrm{mg} \mathrm{kg}^{-1}$ & $\begin{array}{c}\downarrow \text { CAT (brain, liver, } \\
\text { kidney) } \\
\uparrow \text { GSH (liver) } \\
\uparrow S O D \text { (brain) } \\
\downarrow \text { SOD (kidney, liver) } \\
\uparrow \text { MDA (brain, liver, } \\
\text { kidney) }\end{array}$ & $\begin{array}{l}\text { Abarikwu } \\
\quad(73)\end{array}$ \\
\hline
\end{tabular}




\begin{tabular}{|c|c|c|c|c|c|}
\hline Experimental model & $\begin{array}{c}\text { Route of } \\
\text { administration }\end{array}$ & $\begin{array}{c}\text { Exposure } \\
\text { duration }\end{array}$ & $\begin{array}{c}\text { Concentration } \\
\text { / dose }\end{array}$ & $\begin{array}{c}\begin{array}{c}\text { Oxidative stress } \\
\text { markers }\end{array} \\
\end{array}$ & Ref. \\
\hline Male Wistar rats & $\begin{array}{c}\text { orally } \\
\text { administered }\end{array}$ & 15 days & $0.3 \mathrm{mg} \mathrm{kg}^{-1}$ & $\begin{array}{l}\text {-CAT (liver and kidney) } \\
\text { - GPx (liver and kidney) } \\
\text { - GSH (liver and kidney) } \\
\text { - SOD (liver and kidney) } \\
\text {-MDA (liver and } \\
\text { kidney) }\end{array}$ & $\begin{array}{l}\text { Jestadi et al. } \\
\quad(74)\end{array}$ \\
\hline Male Wistar rats & $\begin{array}{c}\text { orally } \\
\text { administered }\end{array}$ & 15 days & $0.3 \mathrm{mg} \mathrm{kg}^{-1}$ & $\begin{array}{l}\text { - CAT (testis) } \\
\text { - GPx (testis) } \\
\text { - GSH (testis) } \\
\text {-GST (testis) } \\
\text { - SOD (testis) } \\
\text {-MDA (testis) } \\
\end{array}$ & $\begin{array}{l}\text { Jestadi et al. } \\
\quad(75)\end{array}$ \\
\hline Female Wistar rats & gavage & 28 days & $\begin{array}{c}5 \mathrm{mg} \mathrm{kg}^{-1} \\
25 \mathrm{mg} \mathrm{kg}^{-1} \\
125 \mathrm{mg} \mathrm{kg}^{-1}\end{array}$ & $\begin{array}{c}\downarrow \text { CAT }\left(125 \mathrm{mg} \mathrm{kg}^{-1}\right) \\
\downarrow \text { GPx }\left(5,125 \mathrm{mg} \mathrm{kg}^{-1}\right) \\
\bullet \text { SOD } \\
\uparrow \operatorname{MDA}\left(125 \mathrm{mg} \mathrm{kg}^{-1}\right)\end{array}$ & $\begin{array}{l}\text { Liu et al. } \\
\quad(76)\end{array}$ \\
\hline Male Wistar rats & gavage & $\begin{array}{l}3 \text { times a } \\
\text { week } / 52 \\
\text { days }\end{array}$ & $12.5 \mathrm{mg} \mathrm{kg}^{-1}$ & $\begin{array}{l}\text { - CAT (testis and liver) } \\
\text { - GPx (testis and liver) } \\
\text { - GSH (testis and liver) } \\
\text { - GST (testis and liver) } \\
\text { - SOD (testis and liver) } \\
\text { - MDA (testis and liver) }\end{array}$ & $\begin{array}{l}\text { Abarikwu et } \\
\text { al. (77) }\end{array}$ \\
\hline Male Wistar rats & gavage & 21 days & $\begin{array}{l}50 \mathrm{mg} \mathrm{kg}^{-1} \\
100 \mathrm{mg} \mathrm{kg}^{-1} \\
300 \mathrm{mg} \mathrm{kg}^{-1}\end{array}$ & $\begin{array}{c}\downarrow \mathrm{CAT}\left(100,300 \mathrm{mg} \mathrm{kg}^{-1}\right. \\
\text { epididymis) } \\
\uparrow \mathrm{CAT}\left(300 \mathrm{mg} \mathrm{kg}{ }^{-1}\right. \\
\text { testis) } \\
\uparrow \mathrm{GPx} \text { (epididymis) } \\
\downarrow \mathrm{GPx}\left(100,300 \mathrm{mg} \mathrm{kg}^{-1}\right. \\
\text { testis) } \\
\uparrow \mathrm{GSH}\left(300 \mathrm{mg} \mathrm{kg}{ }^{-1}\right. \\
\text { epididymis; } 100, \\
300 \mathrm{mg} \mathrm{kg}^{-1} \text { testis) } \\
\downarrow \mathrm{GST}(300 \mathrm{mg} \mathrm{kg} \\
\text { epididymis; } 100, \\
300 \mathrm{mg} \mathrm{kg}^{-1} \text { testis) } \\
\uparrow \mathrm{SOD} \text { (100, 300 mg kg-1} \\
\text { epididymis) } \\
\downarrow \text { SOD (testis) } \\
\bullet \text { MDA (epididymis, } \\
\text { testis) }\end{array}$ & $\begin{array}{l}\text { Abarikwu et } \\
\text { al. (78) }\end{array}$ \\
\hline Adult male albino rats & gavage & 28 days & $300 \mathrm{mg} \mathrm{kg}^{-1}$ & $\begin{array}{l}\downarrow \mathrm{CAT} \\
\downarrow \mathrm{GPx} \\
\downarrow \mathrm{GSH} \\
\downarrow \mathrm{SOD} \\
\uparrow \mathrm{MDA}\end{array}$ & $\begin{array}{l}\text { Abass et al. } \\
\text { (79) }\end{array}$ \\
\hline Male Wistar rats & NA & 10 days & $25 \mathrm{mg} \mathrm{kg}^{-1}$ & $\begin{array}{l}\downarrow \mathrm{CAT} \\
\downarrow \mathrm{GSH} \\
\downarrow \mathrm{SOD} \\
\uparrow \mathrm{MDA}\end{array}$ & $\begin{array}{l}\text { Adedosu et } \\
\text { al. (80) }\end{array}$ \\
\hline
\end{tabular}

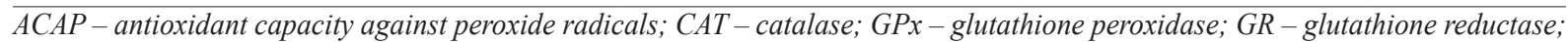
GSH - reduced glutathione; GSSG - oxidised glutathione; GST-glutathione S-transferase; LPO - lipid peroxidation levels; MDA - malondialdehyde; ROS - reactive oxygen species; SOD - superoxide dismutase; TAA - total antioxidant activities; TBARS thiobarbituric acid reactive species

- - no change in oxidative stress marker; $\uparrow$ - increase; $\downarrow$-decrease 
GSH and the activities of CAT, SOD, GPx, and GST (74, 75, 77).

\section{INVOLVEMENT OF OXIDATIVE STRESS IN TERBUTHYLAZINE TOXICITY}

Terbuthylazine (N2-tert-butyl-6-chloro-N4-ethyl-1,3,5triazine-2,4-diamine) has become the key triazine in Europe in the last two decades (37). It is used on a variety of crops, such as cereals, legumes, and under fruit trees as a selective pre- and early post-emergence control agent against most grasses and many annual broadleaf weeds. It is also used as an aquatic herbicide to control submerged and free floating weeds and algae in water courses, reservoirs, and fish ponds (84).

According to the European Food Safety Agency (EFSA), it poses a high risk to non-target plants in the offfield areas, while the risk for bees, soil micro- and macroorganisms, and organisms used in biological wastewater treatment is low (85). EFSA has also warned about high toxicity of both terbuthylazine and its metabolite desethylterbuthylazine to aquatic organisms. In mammals exposed through the oral, dermal, or inhalation routes its acute toxicity can be low to moderate and cause a slight eye and skin irritation and sensitisation. Short-term exposure may affect body weight and food consumption in rats, mice, dogs, and rabbits. Long-term exposure may further affect organ weights in rats and mice and haematological parameters in rats (85).

According to the current cancer classifications, terbuthylazine belongs to Group D - "Not Classifiable as to Human Carcinogenicity" (86). There are several routes of human exposure to terbuthylazine. Occupational exposure occurs through inhalation and dermal contact at workplaces and commercial/industrial settings where terbuthylazine is produced or used. The general population is mostly exposed through ingestion of contaminated drinking water and dermal contact (87). Terbuthylazine has an extensive metabolic pathway. Its major degradation products (desethyl, hydroxy, and desethyl-hydroxyterbuthylazine) have been identified in groundwater above the drinking water limit of $0.1 \mu \mathrm{g} \mathrm{L}-1$ (85).

This herbicide persists in the environment and has a tendency to easily move from treated soils to water compartments through runoffs and leaching $(88,89)$. This has raised concern about its potential toxic effects on aquatic organisms. Table 2 summarises the findings about the affected markers of oxidative stress reported in recent studies on aquatic organisms. However, even though this is a relevant topic, considering that levels as low as $0.1 \mu \mathrm{g} \mathrm{L}^{-1}$ of terbuthylazine and its metabolites in freshwater pose a risk for non-targeted aquatic species, the number of such studies is scarce. Since 2010, only a few studies have been published on common carp, zebrafish, the red swamp crayfish (Procambarus clarkii), and marbled crayfish
(Procambarus fallax $f$. virginalis) (45, 47, 90-97). Most investigated sub-chronic exposure to terbuthylazine at environmentally relevant concentrations and compared them with exposure to higher concentrations (70$\left.1000 \mu \mathrm{g} \mathrm{L}^{-1}\right)$. A 28-day exposure of zebrafish to the environmentally relevant concentration of $0.55 \mu \mathrm{g} \mathrm{L}^{-1}$ of terbuthylazine did not affect oxidative stress biomarkers, while moderate to high concentrations $\left(>400 \mu \mathrm{g} \mathrm{L}^{-1}\right)$ caused a significant decrease in TBARS and GR activity and increase in GST (93). The same was noticed in common carp exposed to $0.38 \mu \mathrm{g} \mathrm{L}-1$ for 91 days, as terbuthylazine did not affect the GSH levels or GST activity (92).

Beside exposure to terbuthylazine or its commercial preparation Click $500 \mathrm{SC}$, aquatic experimental models were exposed to terbuthylazine degradation products, because they pose an even greater risk of water contamination due to high polarity (99). Stara et al. (98) reported higher levels of TBARS and changes in the activities of SOD, CAT, and GR in red swamp crayfish after a 14-day exposure to the environmentally relevant terbuthylazine-desethyl concentration of $2.9 \mu \mathrm{g} \mathrm{L}^{-1}$ (reported in Czech rivers) and the 200 times higher concentration $\left(580 \mu \mathrm{g} \mathrm{L}^{-1}\right)$.

Several studies investigated the effects of terbuthylazine and its degradation products on developing fish (embryos and larvae) under laboratory conditions. Hostovsky et al. (90) reported higher GR activity only when the common carp embryos and larvae were exposed to the higher terbuthylazine concentration $\left(520 \mu \mathrm{g} \mathrm{L}^{-1}\right)$ for 30 days. In contrast, Velisek et al. (94) reported lower SOD activity even from exposure to the lowest concentration of terbuthylazine2-hydroxy $\left(2.9 \mu \mathrm{g} \mathrm{L}^{-1}\right)$, whereas CAT and GR activities were not affected. Similar results for SOD were reported for a 31-day terbuthylazine-desethyl exposure starting with $1.8 \mu \mathrm{g} \mathrm{L}^{-1}$, while no significant difference was found in TBARS, CAT, and GR (95). The same group of authors also investigated the effects of environmentally relevant concentrations of terbuthylazine 2-hydroxy $\left(0.73 \mu \mathrm{g} \mathrm{L}^{-1}\right)$ and terbuthylazine-desethyl $\left(1.80 \mu \mathrm{g} \mathrm{L}^{-1}\right)$ alone and in combination (97). After 36 days of exposure, terbuthylazine-desethyl lowered the SOD and GR activities in embryos and larvae of common carp, whereas terbuthylazine 2-hydroxy and their combination showed no effects. TBARS, CAT, GST, and GSH were not affected at all. In another Velisek et al. study (47), the same metabolites alone or in combination caused a significant increase in CAT activity developing marbled crayfish exposed for 77 days, whereas TBARS levels and SOD and GR activities remained in control range. High concentrations of terbuthylazine-2-hydroxy (375 and $750 \mu \mathrm{g} \mathrm{L}^{-1}$ ) resulted in a significant decrease in TBARS levels and SOD activity after 62 days of exposure of marbled crayfish, but did not significantly affect CAT and GR activity (96).

Very little is known about effects of terbuthylazine on oxidative stress parameters and antioxidant defence in mammals. Recently, we reported that oral 28-day exposure to $0.004,0.4$, and $2.9 \mathrm{mg} \mathrm{kg}^{-1} \mathrm{bw} \mathrm{day}^{-1}$ of terbuthylazine disturbed the oxidant/antioxidant balance in Wistar rats (100). Namely, 
total antioxidant capacity, expressed as plasma FRAP, significantly increased at 0.004 and $0.4 \mathrm{mg} \mathrm{kg}^{-1}$ bw day ${ }^{-1}$. Plasma SOD dropped at 0.004 and $0.4 \mathrm{mg} \mathrm{kg}^{-1}$ bw day ${ }^{-1}$, and plasma CAT at $2.9 \mathrm{mg} \mathrm{kg}^{-1}$ bw day ${ }^{-1}$, whereas erythrocyte SOD increased at $2.9 \mathrm{mg} \mathrm{kg}^{-1}$ bw day-1, and whole blood GPx at $0.4 \mathrm{mg} \mathrm{kg}^{-1}$ bw day ${ }^{-1}$. Exposure to terbuthylazine did not induce significant lipid peroxidation.

\section{EPIDEMIOLOGICAL STUDIES}

Several studies in humans reported an imbalance between oxidative stress markers and the antioxidant defences in farmers/pesticide applicators or agricultural workers (7, 100102). However, a thorough search of relevant literature yielded only one epidemiological study of the association between the biomarkers of atrazine exposure and biomarkers of oxidative stress (103), and none about terbuthylazine. In that one study, no association was found between atrazine mercapturate (AM) and MDA, 8-hydroxy-2'-deoxyguanosine (8-OHdG), or 8-isoprostaglandin-F2 (8-isoPGF) in urine samples collected from 30 farmers over five time points of atrazine application. However, when the authors excluded the samples with AM below the detection limit, they established a significant association between AM and 8-OHdG. These biomarkers did not vary significantly across the five time points. The authors noted that the measured biomarkers were immediate or shortterm responses to environmental exposures, with a lag of up to a few days and that the measured metabolite reflected recent exposure, due to its short half-life.

Human exposure to atrazine or terbuthylazine results in a relatively fast urinary excretion of the parent compounds and their dealkylated and conjugated metabolites $(104,105)$. The half-life of atrazine, for example, is only $24-31 \mathrm{~h}(106,107)$. The parent compound is, therefore, generally detected only in minor amounts in the urine, usually after continuous occupational exposure. In one study (108), for example, atrazine urine ranged between 5 to $29 \mu \mathrm{g} \mathrm{L}{ }^{-1}$ after seven months of continuous work in atrazine production. In another study in corn farmers from Iowa, USA (109), the level of its metabolite AM in urine collected during crop planting of $1.0 \mu \mathrm{g} \mathrm{g}^{-1}$ of creatinine significantly correlated with the amount of atrazine applied per farmer over one year of $217(47-530) \mathrm{kg}$. AM was also detected in only $23 \%$ of non-farm family members from Iowa, USA, with geometric means of $0.067 \mu \mathrm{g} \mathrm{L}^{-1}$ in fathers, $0.031 \mu \mathrm{g} \mathrm{L}^{-1}$ in mothers, and $0.054 \mu \mathrm{g} \mathrm{L}^{-1}$ in children (110). Interestingly, however, Chevrier et al. (111) detected atrazine $\left(0.12 \mu \mathrm{g} \mathrm{L}^{-1}\right.$, max. $\left.0.52 \mu \mathrm{g} \mathrm{L}^{-1}\right)$ and $\operatorname{AM}\left(0.05 \mu \mathrm{g} \mathrm{L}^{-1}\right.$, max. $\left.0.68 \mu \mathrm{g} \mathrm{L}^{-1}\right)$ in the urine of $5.5 \%$ of 579 pregnant women in France for as long as three years after atrazine was banned in Europe.

Terbuthylazine, in turn, was detected in the hair of all farmers, with median and range of $0.61(0.07-4.64) \mathrm{ng} \mathrm{mg}^{-1}$ of hair, but in only $67 \%$ of rural residents, with median and range of $0.01(<0.01-0.04) \mathrm{ng} \mathrm{mg}^{-1}$ of hair (112).
A number of studies evaluated health effects of atrazine exposure in humans. Most of them were retrospective and relied on the levels of atrazine in drinking water (38, 113-116), pesticide use records (117), or self-reported information (118, 119), which, of course, excluded determination of exposure on individual level. Moreover, these studies could not determine whether the observed outcomes were specific to atrazine or co-exposure to other pesticides or other compounds in the environment. Accurate assessment of human exposure is one of the major challenges to epidemiological studies of health effects from pesticide exposure (109). Exposure in farmers is characterised by large seasonal differences in the type, amount, and frequency of pesticide application, as well as application technique, formulations used, environmental conditions, and personal behaviour of pesticide applicator (120, 121). It is particularly challenging to asses exposure for individuals living in the vicinity of pesticide-treated fields or for the general population and to link it with health effects.

Some experimental studies have raised concern that atrazine can induce cancer in humans. However, epidemiological studies that investigated atrazine-related cancer risk have provided no evidence to support this causal relationship, at least judging by the studies looking for associations between atrazine exposure and non-Hodgkin lymphoma or prostate, ovarian, and breast cancer $(1,10,122)$. The major limitation of these epidemiological studies is their inability to reliably assess past exposures. Furthermore, there is no biomarker so specific to reliably establish the link between a certain pesticide and a disease endpoint, which remains the challenge for future studies.

\section{CONCLUSIONS}

Triazines and their residues have been reported in various environmental media, including food and water. Furthermore, s-triazines have been identified as substances hazardous to the aquatic environment and have been included in the EU Priority Pollutants List (123). However, current knowledge about triazine toxicity is still inconclusive.

From the knowledge summarised in Table 1 we can conclude that atrazine generally does not affect lipid peroxidation in developing and adult aquatic organisms, but does set in motion the antioxidant defences (CAT, SOD, GPx, and GST) at low, environmentally relevant concentrations. These defences, however, sometimes cannot compensate for stress at high atrazine concentrations.

Literature reports on aquatic organisms summarised in Table 2 suggest that environmentally relevant concentrations of terbuthylazine do not generally affect the oxidant/ antioxidant balance, but its metabolites do.

In other environmentally relevant organisms, such as drosophila and honeybees, atrazine turned out to be more of a concern because of evident lipid peroxidation and antioxidant depletion. 
Table 2 Changes in oxidative stress markers in experimental models after exposure to terbuthylazine and its metabolites terbuthylazine2-hydroxy and terbuthylazine-desethyl

\begin{tabular}{|c|c|c|c|c|}
\hline $\begin{array}{l}\text { Compound / } \\
\text { concentration }\end{array}$ & Experimental model & $\begin{array}{l}\text { Exposure } \\
\text { duration }\end{array}$ & Oxidative stress markers & Ref. \\
\hline \multicolumn{5}{|l|}{ Terbuthylazine } \\
\hline $\begin{array}{l}0.9 \mu \mathrm{g} \mathrm{L}^{-1} \\
160 \mu \mathrm{g} \mathrm{L}^{-1} \\
520 \mu \mathrm{g} \mathrm{L}^{-1} \\
820 \mu \mathrm{g} \mathrm{L}^{-1}\end{array}$ & $\begin{array}{c}\text { Fish - common carp, embryos } \\
\text { and larvae }\end{array}$ & 30 days & $\begin{array}{c}\uparrow \mathrm{GR}\left(520 \mu \mathrm{g} \mathrm{L}^{-1}\right) \\
\bullet \mathrm{GST} \\
\bullet \mathrm{TBARS}\end{array}$ & $\begin{array}{l}\text { Hostovsky et al. } \\
\text { (90) }\end{array}$ \\
\hline $\begin{array}{l}0.38 \mu \mathrm{g} \mathrm{L}^{-1} \\
60 \mu \mathrm{g} \mathrm{L}^{-1} \\
550 \mu \mathrm{g} \mathrm{L}^{-1}\end{array}$ & Fish - common carp & 91 days & $\begin{array}{c}\bullet \text { GSH } \\
\bullet \text { GST } \\
\uparrow \mathrm{FRAP}\left(550 \mu \mathrm{g} \mathrm{L}^{-1}\right)\end{array}$ & $\begin{array}{l}\text { Mikulikova et al. } \\
\text { (91) }\end{array}$ \\
\hline $3300 \mu \mathrm{g} \mathrm{L}^{-1}$ & Fish - common carp & 24 hours & $\bullet$ FRAP & $\begin{array}{l}\text { Mikulikova et al. } \\
\text { (92) }\end{array}$ \\
\hline $\begin{array}{l}0.55 \mu \mathrm{g} \mathrm{L}^{-1} \\
150 \mu \mathrm{g} \mathrm{L}^{-1} \\
400 \mu \mathrm{g} \mathrm{L}^{-1} \\
700 \mu \mathrm{g} \mathrm{L}^{-1} \\
1000 \mu \mathrm{g} \mathrm{L}^{-1}\end{array}$ & Fish - zebrafish & 28 days & $\begin{array}{c}\bullet \mathrm{GPx} \\
\downarrow \mathrm{GR}\left(700,1000 \mu \mathrm{g} \mathrm{L}^{-1}\right) \\
\uparrow \mathrm{GST}\left(400,700,1000 \mu \mathrm{g} \mathrm{L}^{-1}\right) \\
\downarrow \text { TBARS }\left(1000 \mu \mathrm{g} \mathrm{L}^{-1}\right)\end{array}$ & $\begin{array}{l}\text { Plhalova et al. } \\
\qquad(93)\end{array}$ \\
\hline
\end{tabular}

\begin{tabular}{|c|c|c|c|c|}
\hline \multicolumn{5}{|c|}{ Terbuthylazine-2-hydroxy } \\
\hline $\begin{array}{l}2.9 \mu \mathrm{g} \mathrm{L}^{-1} \\
70 \mu \mathrm{g} \mathrm{L}^{-1} \\
1400 \mu \mathrm{g} \mathrm{L}^{-1} \\
3500 \mu \mathrm{g} \mathrm{L}^{-1}\end{array}$ & $\begin{array}{c}\text { Fish - common carp, embryos } \\
\text { and larvae }\end{array}$ & 35 days & $\begin{array}{l}\bullet \mathrm{CAT} \\
\bullet \mathrm{GR} \\
\downarrow \mathrm{SOD}\end{array}$ & $\begin{array}{l}\text { Velisek et al. } \\
\qquad(94)\end{array}$ \\
\hline $0.73 \mu \mathrm{g} \mathrm{L}^{-1}$ & $\begin{array}{c}\text { Fish - common carp, embryos } \\
\text { and larvae }\end{array}$ & 36 days & $\begin{array}{l}\bullet \mathrm{CAT} \\
\bullet \mathrm{GSH} \\
\bullet \mathrm{GST} \\
\bullet \mathrm{TBARS}\end{array}$ & $\begin{array}{l}\text { Velisek et al. } \\
\qquad(95)\end{array}$ \\
\hline $0.73 \mu \mathrm{g} \mathrm{L}^{-1}$ & Marbled crayfish eggs & 77 days & $\begin{array}{c}\bullet \text { CAT } \\
\bullet \text { GR } \\
\bullet \text { SOD } \\
\bullet \text { TBARS }\end{array}$ & $\begin{array}{l}\text { Velisek et al. } \\
\qquad(47)\end{array}$ \\
\hline $\begin{array}{l}0.75 \mu \mathrm{g} \mathrm{L}^{-1} \\
75 \mu \mathrm{g} \mathrm{L}^{-1} \\
375 \mu \mathrm{g} \mathrm{L}^{-1} \\
750 \mu \mathrm{g} \mathrm{L}^{-1}\end{array}$ & Marbled crayfish eggs & 62 days & $\begin{array}{c}\downarrow \mathrm{SOD}\left(375,750 \mu \mathrm{g} \mathrm{L}^{-1}\right) \\
\bullet \mathrm{CAT} \\
\bullet \mathrm{GR} \\
\downarrow \text { TBARS }\left(375,750 \mu \mathrm{g} \mathrm{L}^{-1}\right)\end{array}$ & $\begin{array}{l}\text { Koutnik et al. } \\
\qquad(96)\end{array}$ \\
\hline \multicolumn{5}{|c|}{ Terbuthylazine-desethyl } \\
\hline $\begin{array}{l}1.80 \mu \mathrm{g} \mathrm{L}^{-1} \\
180 \mu \mathrm{g} \mathrm{L}^{-1} \\
900 \mu \mathrm{g} \mathrm{L}^{-1} \\
1800 \mu \mathrm{g} \mathrm{L}^{-1}\end{array}$ & $\begin{array}{c}\text { Fish - common carp, embryos } \\
\text { and larvae }\end{array}$ & 31 days & $\begin{array}{c}\bullet \mathrm{CAT} \\
\bullet \mathrm{GR} \\
\downarrow \mathrm{SOD} \\
\bullet \mathrm{TBARS}\end{array}$ & $\begin{array}{l}\text { Velisek et al. } \\
\qquad(97)\end{array}$ \\
\hline $1.80 \mu \mathrm{g} \mathrm{L}^{-1}$ & $\begin{array}{c}\text { Fish - common carp, embryos } \\
\text { and larvae }\end{array}$ & 36 days & $\begin{array}{c}\bullet \mathrm{CAT} \\
\downarrow \mathrm{GR} \\
\bullet \mathrm{GSH} \\
\bullet \mathrm{GST} \\
\downarrow \mathrm{SOD} \\
\bullet \mathrm{TBARS}\end{array}$ & $\begin{array}{l}\text { Velisek et al. } \\
\qquad(95)\end{array}$ \\
\hline $1.80 \mu \mathrm{g} \mathrm{L}^{-1}$ & Marbled crayfish eggs & 77 days & $\begin{array}{c}\uparrow \mathrm{CAT} \\
\bullet \mathrm{GR} \\
\bullet \text { SOD } \\
\bullet \mathrm{TBARS}\end{array}$ & $\begin{array}{l}\text { Velisek et al. } \\
\qquad(47)\end{array}$ \\
\hline
\end{tabular}




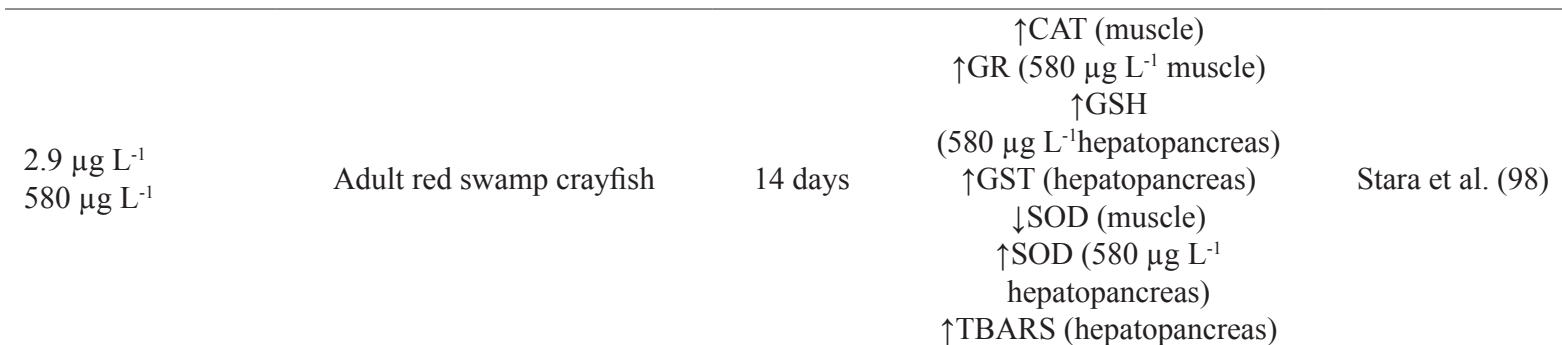

CAT-catalase; FRAP-ferric reducing ability of plasma; GPX-glutathione peroxidase; GR - glutathione reductase; GSH-reduced glutathione; GST - glutathione S-transferase; SOD - superoxide dismutase; TBARS - thiobarbituric acid reactive species $\bullet-$ no change in oxidative stress marker; $\uparrow-$ increase; $\downarrow$-decrease

As for rodents, laboratory studies have confirmed the associations between the two triazines and oxidative stress found in aquatic organisms. They generally suggest that the antioxidant enzyme defences in rodents are compromised.

Although individual biomarkers can be useful as early warning signals, a combination of several markers is more useful to understand the mode of action of a pesticide and identify possible molecular targets. Supporting these notions, the reviewed studies have demonstrated that there is no single specific biomarker of oxidative stress caused by atrazine or terbuthylazine and that a complex approach should be taken to assess oxidative response to pesticides.

\section{Acknowledgements}

This work was financially supported by the project Organic Pollutants in Environment - Markers and Biomarkers of Toxicity (OPENTOX), funded by the Croatian Science Foundation (grant no. 8366).

\section{Conflicts of interest}

None to declare.

\section{REFERENCES}

1. Alavanja MCR, Hoppin JA, Kamel F. Health effects of chronic pesticide Exposure: Cancer and Neurotoxicity. Annu Rev Publ Health 2004;25:155-97. doi: 10.1146/annurev. publhealth.25.101802.123020

2. McCauley LA, Kent Anger W, Keifer M, Langley R, Robson MG, Rohlman D. Studying health outcomes in farmworker populations exposed to pesticides. Environ Health Persp 2006;114:953-60. doi: 10.1289/ehp.8526

3. Mostafalou S, Abdollah M. Pesticides and human chronic diseases: evidences, mechanisms, and perspectives. Toxicol Appl Pharm 2013;268:157-77. doi: 10.1016/j. taap.2013.01.025

4. Abdollahi M, Ranjbar A, Shadnia S, Nikfar S, Rezaie A. Pesticides and oxidative stress: a review. Med Sci Monit 2004;10:RA141-7. PMID: 15173684

5. Soltaninejad K, Abdollahi M. Current opinion on the science of organophosphate pesticides and toxic stress: a systematic review. Med Sci Monit 2009;15:RA75-90. PMID: 19247260

6. Barry KH, Koutros S, Berndt SI, Andreotti G, Hoppin JA, Sandler DP, Burdette LA, Yeager M, Freeman LE, Lubin JH, Ma X, Zheng T, Alavanja MC. Genetic variation in base excision repair pathway genes, pesticide exposure, and prostate cancer risk. Environ Health Persp 2011;119:1726-32. doi: 10.1289/ehp. 1103454

7. Muniz JF, McCauley L, Scherer J, Lasarev M, Koshy M, Kow YW, Nazar-Stewart V, Kisby GE. Biomarkers of oxidative stress and DNA damage in agricultural workers: A pilot study. Toxicol Appl Pharm 2008;227:97-107. doi: 10.1016/j.taap.2007.10.027

8. Ziech D, Franco R, Georgakilas AG, Georgakila S, MalamouMitsi V, Schoneveld O, Pappa A, Panayiotidis MI. The role of reactive oxygen species and oxidative stress in environmental carcinogenesis and biomarker development. Chem-Biol Interact 2010;188:334-9. doi: 10.1016/j. cbi.2010.07.010

9. Simoinello MF, Kleinsorge EC, Scagnetti JA, Mastandrea C, Grigolato RA, Paonessa AM, Carballo MA. Biomarkers of cellular reaction to pesticide exposure in rural population. Biomarkers 2010;15:52-60. doi: 10.3109/13547500903276378

10. Alavanja MC, Ross MK, Bonner MR. Increased cancer burden among pesticide applicators and others due to pesticide exposure. CA Cancer J Clin 2013;63:120-42. doi: 10.3322/caac. 21170

11. Slaninova A, Smutna M, Modrá H, Svobodová Z. A review: oxidative stress in fish induced by pesticides. Neuro Endocrinol Lett 2009;30(Suppl 1):2-12. PMID: 20027135

12. Agrawal A, Bechan Sharma B. Pesticides induced oxidative stress in mammalian systems. Int J Biol Med Res 2010;1:90104.

13. D'Souza UJA. Pesticide toxicity and oxidative stress: a review. Borneo J Med Sci 2017;11:9-19.

14. de Zwart LL, Meerman JH, Commandeur JN, Vermeulen NP. Biomarkers of free radical damage: Applications in experimental animals and in humans. Free Radical Bio Med 1999;26:202-26. doi: 10.1016/S0891-5849(98)00196-8

15. Niedernhofer LJ, Daniels JS, Rouzer CA, Greene RE, Marnett LJ. Malondialdehyde, a product of lipid peroxidation, is mutagenic in human cells. J Biol Chem 2003;278:31426-33. doi: $10.1074 /$ jbc.M212549200

16. Pamplona R. Advanced lipoxidation end-products. ChemBiol Interact 2011;192:14-20. doi: 10.1016/j.cbi.2011.01.007

17. Valko M, Leibfritz D, Moncol J, Cronin MTD, Mazur M, Telser J. Free radicals and antioxidants in normal physiological functions and human disease. Int J Biochem Cell B 2007;39:44-84. doi: 10.1016/j.biocel.2006.07.001

18. Matés JM. Efffects of antioxidant enzymes in the molecular control of reactive oxygen species toxicology. Toxicology 2000;153:83-104. doi: 10.1016/S0300-483X(00)00306-1

19. Di Giulio RT, Meyer JN. Reactive oxygen species and oxidative stress. In: Di Giulio RT, Hinton DE, editors. The 
Toxicology of Fishes. Boca Raton: CRC Press, Taylor and Francis Group; 2008. p. 273-324.

20. Pisoschi AM, Pop A. The role of antioxidants in the chemistry of oxidative stress: A review. Eur J Med Chem 2015;97:5574. doi: 10.1016/j.ejmech.2015.04.040

21. Kniss AR. Long-term trends in the intensity and relative toxicity of herbicide use. Nat Commun 2017;14865: doi: 10.1038/ncomms 14865

22. Dayan FE, Owens DK, Corniani N, Silva FML, Watson SB, Howell JL, Shaner DL. Biochemical markers and enzyme assays for herbicide mode of action and resistance studies. Weed Sci 2015;63:23-63. doi: 10.1614/WS-D-13-00063.1

23. Trebst A. The mode of action of triazine herbicides in plants. In: LeBaron HM, McFarland JE, Burnside OC, editors. The Triazine Herbicides 50 Years Revolutionizing Agriculture. $1^{\text {st }}$ ed. Oxford: Elsevier; 2008. p. 101-10.

24. LeBaron HM, McFarland JE, Burnside OC. The triazine herbicides: a milestone in the development of weed control technology. In: LeBaron HM, McFarland JE, Burnside OC, editors. The Triazine Herbicides 50 Years Revolutionizing Agriculture. $1^{\text {st }}$ ed. Oxford: Elsevier; 2008. p. 1-12.

25. UK Rapporteur Monograph. Council Directive 91/414/EEC Atrazine. Vol 3, Annex B to the Report and Proposed Decision of the United Kingdom made to the European Commission under Article 7(1) of Regulation 3600/92. Summary, Scientific Evaluation and Assessment, 1996.

26. UK Rapporteur Monograph. Addendum to the Report Proposed Decision of the United Kingdom made to the European Commission under Article 7(1) of Regulation 3600/92. Summary, Scientific Evaluation and Assessment, 2000.

27. UK Rapporteur Monograph. Terbuthylazine: The Report and Proposed Decision of the United Kingdom made to the European Commission under Article 8 of 91/414/EEC. August Draft, 2007.

28. National Registrations Authority for Agricultural and Veterinary Chemicals. The NRA review of atrazine, 1997 [displayed 1 June 2018]. Available at https://apvma.gov.au/ sites/default/files/publication/14331-atrazine-interimreview-report.pdf

29. Australian Pesticides and Veterinary Medicines Authority (APVMA). The Reconsideration of Approvals of the Active Constituent Atrazine, Registrations of Products Containing Atrazine, and Their Associated Labels. Canbera: AVMPA; 2004.

30. World Health Organization, International Agency for Research on Cancer. Some Chemicals that Cause Tumours of the Kidney or Urinary Bladder in Rodents and Some Other Substances. IARC Monographs on the Evaluation of Carcinogenic Risks to Humans. Vol 73. Lyon: IARC; 1999.

31. United States Environmental Protection Agency (US EPA) Atrazine Interim Reregistration Eligibility Decision. Appendix L. Assessment of potential mitigation measures for atrazine. Biological and Economic Analysis Division, Office of Pesticide Programs, 2003.

32. United States Environmental Protection Agency (US EPA). Memorandum. Atrazine: Finalization of Interim Reregistration Eligibility Decision and Completion of Tolerance Reassessment and Reregistration Eligibility Process, 2006[displayed 1 June 2018]. Available at https://archive. epa.gov/pesticides/reregistration/web/pdf/atrazine ired finalization.pdf
33. European Commission. Atrazine SANCO/10496/2003-final. Review report for the active substance atrazine; Finalized in the Standing Committee on the Food Chain and Animal Health at its meeting on 3 October 2003 in support of a decision concerning the non-inclusion of atrazine in Annex I of Directive 91/414/EEC and the withdrawal of authorisation for plant protection products containing this active substance. European Commission Health and Consumer Protection Directorate-General, 2003.

34. Dikshith TSS. Handbook of Chemicals and Safety. Boca Raton (FL): CRC Press; 2010.

35. Pucarević M, Šovljanski R, Lazić S, Marijanović N. Atrazine in groundwater of Vojvodina Province. Water Res 2002;36:5120-6. doi: 10.1016/S0043-1354(02)00245-2

36. Drevenkar V, Fingler S, Mendaš G, Stipičević S, Vasilić Ž. Levels of atrazine and simazine in waters in the rural and urban areas of North-West Croatia. Int J Environ An Ch 2004;84:207-16. doi: 10.1080/0306731031000149679

37. Sass JB, Colangelo A. European Union Bans Atrazine, while the United States negotiates continued use. Int J Occup Env Heal 2006;12:260-7. doi: 10.1179/oeh.2006.12.3.260

38. Inoue-Choi M, Weyer PJ, Jones RR, Booth BJ, Cantor KP, Robien K, Ward MH. Atrazine in public water supplies and risk of ovarian cancer among postmenopausal women in the Iowa Women's Health Study. Occup Environ Med 2016;73:582-7. doi: 10.1136/oemed-2016-103575

39. Huang P, Yang J, Ning J, Wang M, Song Q. Atrazine triggers DNA damage response and induces DNA double-strand breaks in MCF-10A cells. Int J Mol Sci 2015;16:14353-68. doi: 10.3390/ijms 160714353

40. Wetzel LT, Luempert LG, Breckenridge CB, Tisdel MO, Stevens JT, Thakur AK, Extrom PJ, Eldridge JC. Chronic effects of atrazine on estrus and mammary tumor formation in female Sprague-Dawley and Fischer 344 rats. J Toxicol Env Health 1994;43:169-82. doi: $10.1080 / 15287399409531913$

41. De Roos AJ, Zahm SH, Cantor KP, Weisenburger DD, Holmes FF, Burmeister LF, Blair A. Integrative assessment of multiple pesticides as risk factors for non-Hodgkin's lymphoma among men. Occup Environ Med 2003;60:e11. doi: 10.1136/oem.60.9.e11

42. Hoar Zahm S, Weisenburger DD, Cantor KP, Holmes FF, Blair A. Role of the herbicide atrazine in the development of non-Hodgkin's lymphoma. Scand J Work Environ Health 1993;19:108-14. doi: 10.5271/sjweh.1499

43. Donna A, Crosignani P, Robutti F, Betta PG, Bocca R, Mariani N, Ferrario F, Fissi R, Berrino F. Triazine herbicides and ovarian epithelial neoplasms. Scand J Work Environ Health 1989;15:47-53. doi: 10.5271/sjweh.1882

44. MacLennan PA, Delzell E, Sathiakumar N, Myers SL, Cheng H, Grizzle W, Chen VW, Wu XC. Cancer incidence among triazine herbicide manufacturing workers. J Occup Environ Med 2002;44:1048-58. doi: 10.1097/00043764-20021100000011

45. Velisek J, Stara A, Zuskova E. Effect of single and combination of three triazine metabolites at environmental concentrations on early life stages of common carp (Cyprinus carpio L.). Environ Sci Pollut R 2016;23:24289-97. doi 10.1007/s11356-016-7689-7

46. Chromcova L, Blahova J, Plhalova L, Zivna D, Stepanova S, Praskova E, Zelníckova L, Skoric M, Svobodova Z. The effects of atrazine exposure on early life stages of common 
carp (Cyprinus carpio). Neuroendocrinol Lett 2013;34(Supp 2):95-101. PMID: 24362100

47. Velisek J, Stara A, Zuskova E, Kouba A. Effects of three triazine metabolites and their mixture at environmentally relevant concentrations on early life stages of marbled crayfish (Procambarus fallax f. virginalis). Chemosphere 2017;175:440-5. doi: 10.1016/j.chemosphere.2017.02.080

48. Adeyemi JA, da Cunha Martins A, Barbosa F. Teratogenicity, genotoxicity and oxidative stress in zebrafish embryos (Danio rerio) co-exposed to arsenic and atrazine. Comp Biochem Phys C 2015;172-173:7-12. doi: 10.1016/j.cbpc.2015.04.001

49. Xing H, Li S, Wang Z, Gao X, Xu S, Wang X. Oxidative stress response and histopathological changes due to atrazine and chlorpyrifos exposure in common carp. Pestic Biochem Phys 2012;103:74-80. doi: 10.1016/j.pestbp.2012.03.007

50. Xing H, Li S, Wang Z, Gao X, Xu S, Wang X. Histopathological changes and antioxidant response in brain and kidney of common carp exposed to atrazine and chlorpyrifos. Chemosphere 2012;88:377-83. doi: $10.1016 / j$. chemosphere.2012.02.049

51. Wang X, Xing H, Jiang Y, Wu H, Sun G, Xu Q, Xu S. Accumulation, histopathological effects and response of biochemical markers in the spleens and head kidneys of common carp exposed to atrazine and chlorpyrifos. Food Chem Toxicol 2013;62:148-58. doi: 10.1016/j.fct.2013.08.044

52. Jin Y, Zhang X, Shu L, Chen L, Sun L, Qian H, Liu W, Fu Z. Oxidative stress response and gene expression with atrazine exposure in adult female zebrafish (Danio rerio). Chemosphere 2010;78:846-52. doi: 10.1016/j. chemosphere.2009.11.044

53. Zhu LS, Shao B, Song Y, Xie H, Wang J, Wang JH, Liu W, Hou XX. DNA damage and effects on antioxidative enzymes in zebra fish (Danio rerio) induced by atrazine. Toxicol Mech Method 2011;21:31-6. doi: 10.3109/15376516.2010.529186

54. Blahová J, Plhalová L, Hostovsky' M, Divišová L, Dobšíková R, Mikulíková I, Stěpánová S, Svobodová Z. Oxidative stress responses in zebrafish Danio rerio after subchronic exposure to atrazine. Food Chem Toxicol 2013;61:82-5. doi: 10.1016/j. fct.2013.02.041

55. Shukla S, Jhamtania RC, Dahiyab MS, Agarwala R. Oxidative injury caused by individual and combined exposure of neonicotinoid, organophosphate and herbicide in zebrafish. Toxicol Rep 2017;4:240-4. doi: 10.1016/j.toxrep.2017.05.002

56. Paulino MG, Souza NES, Fernandes MN. Subchronic exposure to atrazine induces biochemical and histopathological changes in the gills of a Neotropical freshwater fish, Prochilodus lineatus. Ecotox Environ Saf 2012;80:6-13. doi: 10.1016/j.ecoenv.2012.02.001

57. Santos TG, Martinez CBR. Atrazine promotes biochemical changes and DNA damage in a Neotropical fish species. Chemosphere 2012;89:1118-25. doi: 10.1016/j.chemosphere.2012.05.096

58. Mela M, Guiloski IC, Doria HB, Randi MAF, de Oliveira Ribeiro CA, Pereira L, Maraschi AC, Prodocimo V, Freire CA, Silva de Assis HC. Effects of the herbicide atrazine in neotropical catfish (Rhamdia quelen). Ecotox Environ Saf 2013;93:13-21. doi: 10.1016/j.ecoenv.2013.03.026

59. Nwani CD, Lakra WS, Nagpure NS, Kumar R, Kushwaha B, Srivastava SK. Toxicity of the herbicide atrazine: effects on lipid peroxidation and activities of antioxidant enzymes in the freshwater fish Channa punctatus (Bloch). Int J Env Res Pub He 2010;7:3298-312. doi: 10.3390/ijerph7083298
60. Owolabi OD, Omotosho JS. Atrazine-mediated oxidative stress responses and lipid peroxidation in the tissues of Clarias gariepinus. Iran J Toxicol 2017;11:29-38. doi: 10.29252/arakmu.11.2.29

61. Demirci O, Güven K, Asma D, Öğüt S, Uğurlu P. Effects of endosulfan, thiamethoxam, and indoxacarb in combination with atrazine on multi-biomarkers in Gammarus kischineffensis. Ecotox Environ Saf 2018;147:749-58. doi: 10.1016/j.ecoenv.2017.09.038

62. Griboff J, Morales D, Bertrand L, Bonansea RI, Monferrán MV, Asis R, Wunderlin DA, Amé MV. Oxidative stress response induced by atrazine in Palaemonetes argentinus: the protective effect of vitamin E. Ecotoxicol Environ Saf 2014;108:1-8. doi: 10.1016/j.ecoenv.2014.06.025

63. Figueira FH, De Aguiar LM, Da Rosa CE. Embryo-larval exposure to atrazine reduces viability and alters oxidative stress parameters in Drosophila melanogaster. Comp Biochem Phys C 2017;191:78-85. doi: 10.1016/j. cbpc.2016.09.008

64. Helmer SH, Kerbaol A, Aras P, Jumarie C, Boily M. Effects of realistic doses of atrazine, metolachlor, and glyphosate on lipid peroxidation and diet-derived antioxidants in caged honeybees (Apis mellifera). Environ Sci Pollut R 2015;22:8010-21. doi: 10.1007/s11356-014-2879-7

65 . Williams JR. Biomarkers of oxidative stress in atrazinetreated honeybees: a laboratory and in-hive study. $[\mathrm{PhD}$ thesis]. Blacksburg: Faculty of the Virginia Polytechnic Institute and State University; 2016.

66. EL-Shenawy NS, El-Ahmary B, Al-Eisa RA. Mitigating effect of ginger against oxidative stress induced by atrazine herbicides in mice liver and kidney. J Biofertil Biopestici 2011;2:107. doi: 10.4172/2155-6202.1000107

67. Gao S, Wang Z, Zhang C, Jia L, Zhang Y. Oral exposure to atrazine induces oxidative stress and calcium homeostasis disruption in spleen of mice. Oxid Med Cell Longev 2016;ID 7978219. doi: 10.1155/2016/7978219

68. Singh M, Sandhir R, Kiran R. Oxidative stress induced by atrazine in rat erythrocytes: mitigating effect of vitamin E. Toxicol Mech Method 2010;20:119-26. doi: 10.3109/15376511003606253

69. Abarikwu SO, Adesiyan AC, Oyeloja TO, Oyeyemi MO, Farombi EO. Changes in sperm characteristics and induction of oxidative stress in the testis and epididymis of experimental rats by a herbicide, atrazine. Arch Environ Con Tox 2010;58:874-82. doi: 10.1007/s00244-009-9371-2

70. Singh M, Sandhir R, Kiran R. Effects on antioxidant status of liver following atrazine exposure and its attenuation by vitamin E. Exp Toxicol Pathol 2011;63:269-76. doi: 10.1016/j.etp.2010.01.005

71. Pogrmić-Majkić K, Kaisarević S, Fa S, Dakić V, Glisić B, Hrubik J, Kovačević R. Atrazine effects on antioxidant status and xenobiotic metabolizing enzymes after oral administration in peripubertal male rat. Environ Toxicol Phar 2012;34:495501. doi: 10.1016/j.etap.2012.06.004

72. Shirisha K, Mastan M. Effect of antioxidant status on liver following atrazine exposure and its protection by Andrographis paniculata. IOSR J Pharm Biol Sci 2013;7:3641.

73. Abarikwu SO. Protective effect of quercetin on atrazine-induced oxidative stress in the liver, kidney, brain, and heart of adult Wistar rats. Toxicol Int 2014;21:148-55. doi: 10.4103/0971-6580.139794 
74. Jestadi DB, Phaniendra A, Babji U, Srinu T, Shanmuganathan B, Periyasamy L. Effects of short term exposure of atrazine on the liver and kidney of normal and diabetic rats. J Toxicol 2014;ID 536759. doi: 10.1155/2014/536759

75. Jestadi DB, Phaniendra A, Babji U, Shanmuganathan B, Periyasamy L. Effects of atrazine on reproductive health of nondiabetic and diabetic male rats. Int Sch Res Notices 2014;ID 676013. doi: 10.1155/2014/676013

76. Liu W, Du Y, Liu J, Wang H, Sun D, Liang D, Zhao L, Shang $\mathrm{J}$. Effects of atrazine on the oxidative damage of kidney in Wister rats. Int J Clin Exp Med 2014;7:3235-43. PMCID: PMC4238554

77. Abarikwu SO, Akiri OF, Durojaiye MA, Adenike A. Combined effects of repeated administration of Bretmont Wipeout (glyphosate) and Ultrazin (atrazine) on testosterone, oxidative stress and sperm quality of Wistar rats. Toxicol Mech Method 2015;25:70-80. doi: 10.3109/15376516.2014.989349

78. Abarikwu SO, Duru QC, Chinonso OV, Njoku RC. Antioxidant enzymes activity, lipid peroxidation, oxidative damage in the testis and epididymis, and steroidogenesis in rats after co-exposure to atrazine and ethanol. Andrologia 2016;48:548-57. doi: 10.1111/and.12478

79. Abass MS, Elkhateeb SA, EL-Baset SAA, Kattaia AA, Mohamed EM, Atteia HH. Lycopene ameliorates atrazineinduced oxidative damage in adrenal cortex of male rats by activation of the Nrf2/HO-1 pathway. Environ Sci Pollut R 2016;23:15262-74. doi: 10.1007/s11356-016-6637-x

80. Adedosu O, Badmus A, Adeleke G, Raji R, Akintolaa L. Telfairia occidentalis seed extract protects against oxidative stress, inflammation and some haematological disorders associated with atrazine-induced prostate cancer in rats. Eur J Cancer 2017;72(Suppl 1):S94. doi: 10.1016/S09598049(17)30393-3

81. Torres C, Ribas G, Xamena N, Creus A, Marcos R. Genotoxicity of four herbicides in the Drosophila wing spot test. Mutat Res 1992;280:291-95. doi: 10.1016/01651218(92)90060-D

82. Marcus SR, Fiumera AC. Atrazine exposure affects longevity, development time and body size in Drosophila melanogaster. J Insect Physiol 2016;91-92:18-25. doi: 10.1016/j. jinsphys.2016.06.006

83. Narotsky MG, Best DS, Guidici DL, Cooper RL. Strain comparisons of atrazine-induced pregnancy loss in the rat. Reprod Toxicol 2000;15:61-9. doi: 10.1016/S08906238(00)00111-8

84. World Health Organization (WHO). Terbuthylazine in drinking-water. Background document for preparation of WHO Guidelines for drinking-water quality [displayed 4 Juny 2018]. Available at http:/www.who.int/water_sanitation health/dwq/chemicals/terbuthylazine.pdf

85. European Food Safety Authority (EFSA). Conclusion on the peer review of the pesticide risk assessment of the active substance terbuthylazine, 2011 [displayed 4 Juny 2018]. Available at http:// onlinelibrary.wiley.com/doi/10.2903/j.efsa.2011.1969/epdf

86. USEPA Office of Pesticide Programs. Chemicals Evaluated for Carcinogenic Potential. Annual Cancer Report 2017 [displayed 4 Juny 2018]. Available at http://npic.orst.edu/ chemicals_evaluated.pdf

87. US Environmental Protection Agency (US EPA). Reregistration Eligibility Decisions (REDs) Database on Terbuthylazine (5915 41-3). USEPA 738-R-95-005. [displayed 4 Juny 2018].
Available at https://archive.epa.gov/pesticides/reregistration/web/ pdf/2645.pdf

88. Calderon MJ, De Luna E, Gomez JA, Hermosin MC. Herbicide monitoring in soil, runoff waters and sediments in an olive orchard. Sci Total Environ 2016;(569-570):416-22. doi: 10.1016/j.scitotenv.2016.06.126

89. Stipičević S, Mendaš G, Dvoršćak M, Fingler S, Galzina N, Barić K. Dissipation dynamics of terbuthylazine in soil during the maize growing season. Arh Hig Rada Toksikol 2017;68:336-42. doi: 10.1515/aiht-2017-68-3063

90. Hostovsky M, Blahova J, Plhalova L, Stepanova S, Praskova E, Marsalek P, Svobodova Z. Oxidative stress parameters in early developmental stages of common carp (Cyprinus carpio L.) after subchronic exposure to terbuthylazine and metribuzin. Neuroendocrinol Lett 2012;33(Suppl 3):124-9. PMID: 23353855

91. Mikulíkova I, Modra H, Blahova J, Marsalek P, Groch L, Siroka Z, Kruzikova K, Jarkovsky J, Littnerova S, Svobodova $Z$. The effects of Click $500 \mathrm{SC}$ (terbuthylazine) on common carp Cyprinus carpio under (sub)chronic conditions. Neuroendocrinol Lett 2011;32(Suppl 1):15-24. PMID:22167222

92. Mikulikova I, Modra H, Blahova J, Kruziková K, Marsalek P, Bedanova I, Svobodova Z. Recovery ability of common carp (Cyprinus carpio) after a short-term exposure to terbuthylazine. Pol J Vet Sci 2013;16:17-23. doi: 10.2478/pjvs-2013-0003

93. Plhalova L, Stepanova S, Blahova J, Praskova E, Hostovsky M, Skoric M, Zelnickova L, Svobodova Z, Bedanova I. The effects of subchronic exposure to terbuthylazine on zebrafish. Neuroendocrinol Lett 2012;33(Suppl 3):113-9. PMID:23353853

94. Velisek J, Stara A, Koutnik D, Machova J. Effect of terbuthylazine-2-hydroxy at environmental concentrations on early life stages of Common Carp (Cyprinus carpio L.). Biomed Res Int 2014;ID 621304. doi: 10.1155/2014/621304

95. Koutnik D, Stara A, Zuskova E, Kouba A, Velisek J. The chronic effects of terbuthylazine-2-hydroxy on early life stages of marbled crayfish (Procambarus fallax $\mathrm{F}$. virginalis). Pestic Biochem Phys 2017;136:29-33. doi: 10.1016/j. pestbp.2016.08.008

96. Velisek J, Koutnik D, Zuskova E, Stara A. Effects of the terbuthylazine metabolite terbuthylazine-desethyl on common carp embryos and larvae. Sci Total Environ 2016;539:214-20. doi: 10.1016/j.scitotenv.2015.08.152

97. Stara A, Zuskova E, Kouba A, Velisek J. Effects of terbuthylazine-desethyl, a terbuthylazine degradation product, on red swamp crayfish (Procambarus clarkii). Sci Total Environ 2016;566-567:733-40. doi: 10.1016/j. scitotenv.2016.05.113

98. Loos R, Locoro G, Comero S, Contini S, Schwesig D, Werres F, Balsaa P, Gans O, Weiss S, Blaha L, Bolchi M, Gawlik BM. PanEuropean survey on the occurrence of selected polar organic persistent pollutants in ground water. Water Res 2010;44:4115-26. doi: 10.1016/j.watres.2010.05.032

99. Tariba Lovaković B, Pizent A, Kašuba V, Kopjar N, Micek V, Mendaš G, Dvoršćak M, Mikolić A, Milić M, Žunec S, Lucić Vrdoljak A, Želježić D. Effects of sub-chronic exposure to terbuthylazine on DNA damage, oxidative stress and parent compound/metabolite levelsinadultmalerats. FoodChem Toxicol 2017;108:93-103. doi: 10.1016/j.fct.2017.07.046

100. Kisby GE, Muniz JF, Scherer J, Lasarev MR, Koshy M, Kow YW, McCauley L. Oxidative stress and DNA damage in 
agricultural workers. J Agromedicine 2009;14:206-14. doi: 10.1080/10599240902824042

101. Wafa T, Nadia K, Amel N, Ikbal C, Insaf T, Asma K, Hedi MA, Mohamed H. Oxidative stress, hematological and biochemical alterations in farmers exposed to pesticides. J Environ Sci Heal B 2013;48:1058-69. doi: 10.1080/03601234.2013.824285

102. Lee KM, Park S-Y, Lee K, Oh S-S, Ko SB. Pesticide metabolite and oxidative stress in male farmers exposed to pesticide. Ann Occup Environ Med 2017;29:5 doi: 10.1186/ s40557-017-0162-3

103. Lerro CC, Beane Freeman LE, Portengen L, Kang D, Lee K, Blair A, Lynch CF, Bakke B, De Roos AJ, Vermeulen RCH. A longitudinal study of atrazine and 2,4-D exposure and oxidative stress markers among Iowa corn farmers. Environ Mol Mutagen 2017;58:30-8. doi: 10.1002/em.22069

104. Mendaš G, Vuletić M, Galić N, Drevenkar V. Urinary metabolites as biomarkers of human exposure to atrazine: atrazine mercapturate in agricultural workers. Toxicol Lett 2012;210:171-81. doi: 10.1016/j.toxlet.2011.11.023

105. Barr DB, Panuwet P, Nguyen JV, Udunka S, Needham LL. Assessing exposure to atrazine and its metabolites using biomonitoring. Environ Health Persp 2007;115:1474-8. doi: 10.1289/ehp.10141

106. Gilman SD, Gee SJ, Hammock BD, Vogel JS, Haack K, Buchholz BA, Freeman SP, Wester RC, Hui X, Maibach HI. Analytical performance of accelerator mass spectrometry and liquid scintillation counting for detection of ${ }^{14} \mathrm{C}$-labeled atrazine metabolites in human urine. Anal Chem 1998;70:3463-9. doi: 10.1021/ac971383v

107. Campbell Jr JL, Andersen ME, Hinderliter PM, Yi KD, Pastoor TP, Breckenridge CB, Clewell HJ $3^{\text {rd }}$. PBPK model for atrazine and its chlorotriazine metabolites in rat and human. Toxicol Sci 2016;150:441-53. doi: 10.1093/toxsci/ kfw014

108. Mendaš G, Tkalčević B, Drevenkar V. Determination of chloro- and methylthiotriazine compounds in human urine: extraction with diethyl ether and $\mathrm{C}_{18}$ solid-phase extraction for gas chromatographic analysis with nitrogen-selective and electron capture detection. Analyt Chim Acta 2000;424:7-18. doi: 10.1016/S0003-2670(00)01150-8

109. Bakke B, De Roos AJ, Barr DB, Stewart PA, Blair A, Freeman LB, Lynch CF, Allen RH, Alavanja MCR. Exposure to atrazine and selected non-persistent pesticides among corn farmers during a growing season. J Expo Sci Env Epid 2009;19:544-54. doi: 10.1038/jes.2008.53

110. Curwin BD, Hein MJ, Sanderson WT, Striley C, Heederik D, Kromhout H, Reynolds SJ, Alavanja MC. Urinary pesticide concentrations among children, motjers and fathers living in farm and non-farm households in Iowa. Ann Occup Hyg 2007;51:53-65. doi: 10.1093/annhyg/mel062

111. Chevrier C, limon G, Monfort C, Rouget F, Garlantézec R, Petit C, Durand G, Cordier S. Urinary biomarkers of prenatal atrazine exposure and adverse birth outcomes in the PELAGIE birth cohort. Environ Health Persp 2011;119:103441. doi: 10.1289/ehp.1002775
112. Mercadante R, Polledri E, Giavini E, Menegola E, Bertazzi PA, Fustinoni S. Terbuthylazine in hair as a biomarker of exposure. Toxicol Lett 2012;210:169-73.

113. Villanueva CM, Durand G, Coutté MB, Chevrier C, Cordier $\mathrm{S}$. Atrazine in municipal drinking water and risk of low birth weight, preterm delivery, and small-for-gestational-age status. Occup Environ Med 2005;62:400-5. doi: 10.1136/ oem.2004.016469

114. McElroy JA, Gangnon RE, Newcomb PA, Kanarek MS, Anderson HA, Brook JV, Trentham-Dietz A, Remington PL. Risk of breast cancer for women living in rural areas from adult exposure to atrazine from well water in Wisconsin. J Expo Sci Env Epid 2007;17:207-14. doi: 10.1038/sj. jes.7500511

115. Ochoa-Acuña H, Frankenberger J, Hahn L, Carbajo C. Drinking-water herbicide exposure in Indiana and prevalence of small-for-gestation-age and preterm delivery. Environ Health Persp 2009;117:1619-24. doi: 10.1289/ehp.0900784

116. Cragin LA, Kesner JS, Bachand AM, Barr DB, Meadows JW, Krieg EF, Reif JS. Menstrual cycle characteristics and reproductive hormone levels in women exposed to atrazine in drinking water. Environ Res 2011;111:1293-301. doi: 10.1016/j.envres.2011.09.009

117. Reynolds P, Hurley SE, Goldberg DE, Yerabati S, Gunier RB, Hertz A, Anton-Culver H, Bernstein L, Deapen D, HornRoss PL, Peel D, Pinder R, Ross RK, West D, Wright WE, Ziogas A. Residential proximity to agricultural pesticide use and incidence of breast cancer in the California Teachers Study cohort. Environ Res 2004;96:206-18. doi: 10.1016/j. envres.2004.03.001

118. Rusiecki JA, De Roos A, Lee WJ, Dosemeci M, Lubin JH, Hoppin JA, Blair A, Alavanja MCR. Cancer incidence among pesticide applicators exposed to atrazine in the Agricultural Health Study. JNCI-J Natl Cancer I 2004;96:1375-82. doi: 10.1093/jnci/djh264

119. Beane Freeman LE, Rusiecki JA, Hoppin JA, Lubin JH, Koutros S, Andreotti G, Hoar Zahm S, Hines CJ, Coble JB, Barone-Adesi F, Sloan J, Sandler DP, Blair A, Alavanja MCR. Atrazine and cancer incidence among pesticide applicators in the Agricultural Health Study (1994-2007). Environ Health Persp 2011;119:1253-9. doi: 10.1289/ehp.1103561

120. Kromhout H, Heederik D. Effects of errors in the measurement of agricultural exposures. Scand J Work Env Hea 2005:31(Suppl 1):33-8. PMID:16190147

121. Brouwer M, Kromhout H, Vermeulen R, Duyzer J, Kramer H, Hazeu G, de Snoo G, Huss A. Assessment of residential environmental exposure to pesticides from agricultural fields in the Netherlands. J Expo Sci Env Epid 2018;28:173-81. doi: $10.1038 /$ jes.2017.3

122. Sathiakumar N, MacLennan PA, Mandel J, Delzell E. A review of epidemiological studies of triazine herbicides and cancer. Crit Rev Toxicol 2011;41(Suppl 1):1-34. doi: $10.3109 / 10408444.2011 .554793$

123. European Environment Agency (EEA). Hazardous Substances in Europe's Fresh and Marine Waters. An overview. Technical report 8. Luxembourg: Publications Office of the European Union; 2011. 


\section{Oksidacijski stres u toksičnosti triazinskih pesticida - pregled glavnih biomarkera}

Ovaj pregledni članak daje sažetak studija objavljenih od 2010. godine o djelovanju atrazina i terbutilazina na parametre oksidacijskog stresa (osobito lipidnu peroksidaciju i antioksidacijske enzime) u eksperimentalnih životinja i ljudi. Općenito je utvrđeno da izloženost eksperimentalnih životinja atrazinu i terbutilazinu najviše utječe na njihovu antioksidacijsku obranu i u manjoj mjeri na lipidnu peroksidaciju, ali učinci variraju ovisno o vrsti, spolu, dobi, koncentraciji herbicida i trajanju izlaganja. Većina studija uključivala je vodene organizme kao korisne i osjetljive bio-pokazatelje onečišćenja okoliša i važan dio prehrambenog lanca. U laboratorijskih miševa i štakora promjene u markerima oksidacijskog stresa bile su vidljive samo nakon izloženosti visokim dozama atrazina. U svojem smo nedavnom izvješću iznijeli podatak da niske doze terbutilazina također mogu inducirati oksidacijski stres u Wistar štakora. Očigledno je da pri svakoj eksperimentalnoj procjeni toksičnih učinaka pesticida treba uzeti u obzir kombinaciju nekoliko biomarkera oksidacijskog stresa i antioksidacijske obrane u različitim tkivima i stanicama. Učinci identificirani u eksperimentalnim modelima trebaju se nadopunjavati i potvrditi epidemiološkim istraživanjima. To je važno ako želimo razumjeti utjecaj pesticida na ljudsko zdravlje i uspostaviti sigurnu granicu izloženosti.

KLJUČNE RIJEČI: atrazin; antioksidacijski enzimi; lipidna peroksidacija; terbutilazin 